INRA Prod. Anim.,

2012,25 (2), 141-158

\title{
Conséquences zootechniques de simplifications de pratiques : exemples de la distribution des aliments et de la traite des vaches
}

\author{
J. AGABRIEL ${ }^{1,2}$, J.-P. FARRIE 3 , E. POTTIER 3,4 , P. NOTE5 , D. POMIES 1,2 \\ ${ }^{1}$ INRA, UMR1213 Herbivores, F-63122 Saint-Genès-Champanelle, France \\ 2 Clermont Université, VetAgro Sup, UMR Herbivores, BP 10448, F-63000 Clermont-Ferrand, France \\ ${ }^{3}$ Institut de l'Elevage, 9 Allée Pierre de Fermat, F-63170 Aubière, France \\ ${ }^{4}$ CIIRPO, Le Mourier, F-87800 Saint-Priest-Ligoure, France \\ 5 INRA, UE1296 Monts d'Auvergne, F-63210 Orcival, France \\ Courriel : jacques.agabriel@clermont.inra.fr
}

La simplification des pratiques s'impose souvent dans les élevages compte tenu de l'agrandissement des troupeaux et de l'évolution de la productivité du travail qui l'accompagne. Elle concerne le travail d'astreinte comme l'affouragement, la distribution des concentrés ou la traite. Pour réaliser le projet de l'éleveur les animaux doivent continuer à produire et s'adapter aux changements d'environnement physique, nutritionnel ou social que les pratiques simplifiées induisent. Les risques de baisse des performances animales doivent être maîtrisés à différentes échelles de temps ${ }^{1}$.

La taille des troupeaux et la production par éleveur ont régulièrement augmenté dans les exploitations d'élevage de ruminants au cours de la dernière décennie, au détriment du temps de travail qui s'est fortement accru. Une réflexion approfondie sur les pratiques d'élevage s'est donc vite révélée nécessaire dans ce contexte d'agrandissement. La simplification des pratiques quotidiennes vise à mieux aménager ou à réduire les contraintes du travail c'està-dire sa quantité, sa pénibilité, et son caractère répétitif. Selon les cas, les objectifs d'une simplification peuvent viser davantage à réduire la pénibilité par exemple la traite en atelier laitier, ou à réduire la fréquence des opérations de soins comme l'allotement ou la distribution de la ration. D'après les synthèses récentes des Bilans Travail réalisées par le RMT Travail en élevage, (Cournut et Chauvat 2012), les pratiques qui requièrent le plus de travail de la part des éleveurs portent sur l'alimentation des animaux, plus particulièrement leur affouragement en hiver et, dans le cas des animaux laitiers, sur la traite. Le travail d'astreinte d'un troupeau allaitant de 100 vaches correspond par exemple à $413 \mathrm{~h}$ de travail soit $19 \mathrm{~h}$ par semaine dont $50 \%$ correspond à l'alimentation (Kentzel 2010). La traite biquotidienne d'un troupeau d'une cinquantaine de vaches par un seul trayeur prend environ 3 heures par jour, nettoyage des locaux compris.

Simplifier les pratiques d'élevage des animaux peut induire de fortes variations dans leur environnement nutritionnel et social. Si les lots sont de plus grande taille, les ré-allotements moins fréquents, et les traitements moins individualisés, les animaux peuvent se retrouver en situation de compétition plus intense et risquent des situations de «mal-être» avérées, avec une augmentation significative de la variabilité des performances zootechniques. Ainsi, la distribution classique plusieurs fois par jour des principaux ingrédients de la ration (fourrages et concentrés), vise à maîtriser les quantités distribuées et à réguler les apports. Cette pratique habituelle, et qui sert de témoin dans les expérimentations, permet de faire s'exprimer au mieux le potentiel de production des animaux. A l'opposé, la simplification des pratiques d'alimentation peut réduire l'homogénéité des apports si elle consiste à modifier le rythme de distribution de certains aliments en les espaçant ou en les alternant, ou à les homogénéiser encore davantage si tous les aliments sont regroupés dans une seule ration mélangée.

Les pratiques simplifiées ont un impact sur les fonctions physiologiques de croissance, lactation et reproduction, différent selon les catégories d'animaux, jeunes ou âgés. Elles vont mobiliser la panoplie des réponses adaptatives physiologiques et comportementales des animaux qui ont été décrites (Blanc et al 2010), notamment lorsqu'il s'agit de réponses à court et moyen terme comme pour la lactation (Friggens et al 2010). Mais à l'inverse, la connaissance de ces réponses adaptatives peut être à la base de l'innovation pour de nouvelles simplifications en réduisant au maximum les risques de contre-performances, par l'estimation de l'éventail des possibles. C'est notamment le cas chez la vache allaitante où la maîtrise de la relation nutrition-reproduction par la note d'état corporel permet d'éviter les risques de baisse de

\footnotetext{
${ }^{1}$ Texte proposé et discuté dans le cadre de l’UMT PASF 63122 Saint-Genès-Champanelle.
} 
productivité, comme le montrent les exemples que nous développerons plus loin.

Cet article fait ainsi le point de diverses conséquences mesurables induites par des simplifications choisies de pratiques d'élevage. Nous analyserons les conséquences zootechniques (ingestion, production, croissance) de modifications raisonnées de pratiques de l'alimentation et de la traite pouvant réduire le temps de travail sur ces deux postes. Les pratiques d'alimentation se rapportent à la manière dont se fait la distribution des aliments (fourrages et concentrés) et aux rythmes de leurs distributions à l'échelle de la semaine voire de plusieurs semaines.

Les exemples que nous développons portent sur une large gamme de niveaux de production, et donc entraînant des risques de contre-performances de plus en plus importants et variés au fur et à mesure que ce niveau s'accroît. Nous avons considéré les modifications des performances au niveau de l'individu comme au niveau du groupe (par exemple la baisse de la production de lait d'une vache ou celle du gain moyen quotidien d'une case de taurillons) en considérant, quand cela est possible, également les modifications induites sur la variabilité des performances. Les résultats présentés ont été obtenus en stations expérimentales, dans lesquelles les modifications de pratiques et donc de l'environnement social et nutritionnel sont maîtrisées et résultent d'une réflexion préliminaire de groupes d'experts. Mais elles sont parfois volontairement extrêmes par rapport à des conditions de terrain, avec l'intention de faire exprimer à l'animal des réponses physiologiques et/ou comportementales marquées. A l'inverse, dans les modalités quotidiennes de la mise en œuvre, les expérimentateurs n'ont pas voulu placer les animaux en situations de risque sanitaire. Des conséquences sanitaires graves ne sont donc que peu observées ou rapportées, ce qui ne serait pas obligatoirement le cas en élevage commercial si les mêmes modifications de pratique étaient directement appliquées.

\section{1 / Simplifier la distribution des aliments}

La mise en œuvre de la simplification de la distribution peut s'envisager en tenant compte de la nature des aliments (fourrages et aliments concentrés) et des types d'animaux qui reçoivent les rations. Les exemples qui ont été retenus portent en premier lieu sur des génisses et vaches allaitantes en phase de croissance ou de production. La capacité de ces animaux à supporter des à-coups alimentaires a été largement décrite et repose sur la flexibilité et la plasticité de la dépense d'entretien et sur l'utilisation des réserves corporelles (Petit et Agabriel 1993). Les performances globales à l'échelle du cycle de production ou de la carrière sont peu modifiées grâce aux capacités de compensation de ces animaux lorsqu'arrivent des périodes où l'alimentation est pléthorique.

La distribution des aliments concentrés peut également être simplifiée. Cet aspect a été abordé en élevage ovin allaitant où la finition des agneaux en bergerie nécessite d'importantes quantités de concentrés, ce qui n'est pas toujours compatible avec l'accroissement de leurs prix. L'objectif est alors de réduire la double contrainte du temps de distribution et du coût de la ration, mais également de répondre au souhait souvent exprimé des éleveurs de développer les mélanges fermiers, pour sécuriser l'alimentation par la maîtrise des constituants.

Les conséquences de ces simplifications de pratiques d'alimentation ont été mesurées en conditions expérimentales pour évaluer à la fois l'ingestion et la performance d'utilisation digestive.

\section{1 / Distribuer une ration com- plète mélangée et riche en foin}

Avec des génisses et/ou des vaches allaitantes, plusieurs simplifications de la distribution de l'alimentation hivernale ont ainsi été identifiées. La distribution de tous les aliments - fourrages et concentrés - en un seul passage est possible par l'utilisation d'une remorque mélangeuse dans les systèmes d'alimentation basés à la fois sur de l'ensilage et du foin. Elle permet une mécanisation complète de ce poste de travail et un gain de temps de l'ordre de $35 \%$ du travail d'astreinte.

La ration complète est une pratique largement développée en élevage laitier. Sa composition est calculée pour satisfaire les besoins d'un lot en fonction du potentiel laitier moyen. Les rations dans ces systèmes sont ensuite ajustées individuellement par complémentation en salle de traite (Faverdin et al 2007). Lorsque cette technique est utilisée, elle conduit en pratique à des niveaux d'ingestion légèrement plus élevés que lorsque les apports de fourrages et de concentrés sont séparés, et cela d'autant plus que les fourrages utilisés sont grossiers ou le concentré limité (Keane et Drennan 2006). Elle conduit également à suralimenter les vaches les plus faibles productrices des lots si le mélange est calculé sur la vache «moyenne». Les conséquences d'un tel mode de distribution ont été évaluées en élevage bovin allaitant, avec des rations moins denses en énergie et donc plus riches en fourrages grossiers.

On observe aussi sur le terrain la mise à disposition des fourrages en libre service dans de grands râteliers approvisionnés une fois par semaine, pour les systèmes d'alimentation sans ensilage basés sur des stocks exclusivement conditionnés en balles (foin et/ou enrubannage). Ce deuxième type de pratique a été analysé surtout sur les vaches allaitantes au comportement d'ingestion flexible (Ingrand et Agabriel 1997).

a) Effets de la ration mélangée sur l'ingestibilité des rations à base de foin

Une expérimentation a été mise en place à la Station de Jalogny, par la Chambre d'Agriculture de la Saône et Loire et l'Institut de l'Elevage, dont les principaux résultats, rappelés ici, ont été développés plus largement par Farrié et al (2004). Des lots de génisses Charolaises de deux ans (13 à 16 lots selon les années) ont reçu une ration complète mélangée en un seul passage journalier, au cours de 3 hivers successifs pendant respectivement 56, 59 et 99 jours (figure 1). L'opération de mélange a consisté à brasser et à découper les fibres les plus longues d'un mélange d'ensilage (herbe ou maïs) de foin et de concentré. Les quantités journalières ingérées et les croissances ont été mesurées. Le lot «témoin» a reçu une ration d'ensilage complémentée en céréales ou en tourteaux distribués en quantité fixe. Un fourrage grossier foin (ou paille) était distribué en plus ad libitum, en fin de matinée (environ $10 \%$ de refus). Dans le lot expérimental «mélangé», la ration était composée des mêmes quantités d'ensilage et de concentré que pour le lot «témoin», avec du foin distribué en quantité tel que les refus s'élèvent à 3 - $5 \%$ du distribué d'un jour sur l'autre. Dans les deux cas, l'ajustement des quantités ingérées réalisé par l'animal, portait sur la quantité de foin ingérée.

En moyenne sur les 3 essais, les génisses recevant la ration mélangée ont ingéré 1 à $2 \mathrm{~kg}$ de MS de plus que celles du lot témoin soit $+17 \%$ (figure 1 ). Les niveaux d'énergie ingérée ont donc été supérieurs de 1 UFL $(8,5 v s$ 7,5) entraînant des croissances supérieures des génisses en moyenne de $120 \mathrm{~g} / \mathrm{j}$ (747 au lieu de $628 \mathrm{~g} / \mathrm{j})$. On peut ainsi considérer que ce type de régime a eu comme effet de diminuer la valeur d'encombrement de la ration.

Les couteaux de la mélangeuse réduisent la taille des brins du fourrage, 
Figure 1. Consommations quotidiennes (kg MS) par des génisses Charolaises de 2 ans selon deux modalités d'alimentation ( $X$ : ration mélangée, $T$ : lot témoin distribution séparée) dans une série de trois essais indépendants (Farrié et al 2004).

Les valeurs affichées (kg MS) correspondent aux quantités de foin ou de paille ingérées.

$\mathrm{kg}$ MS

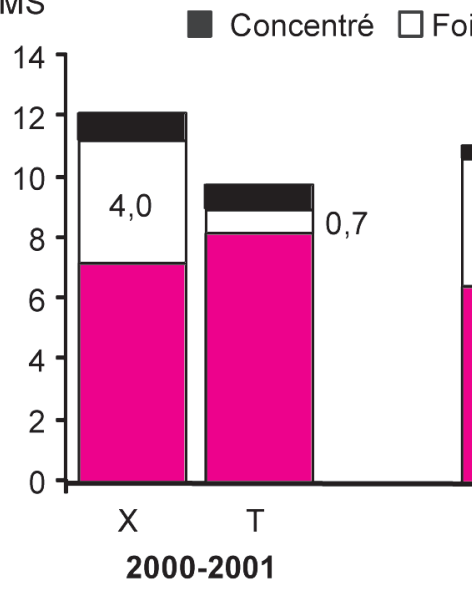

facteur favorable à l'ingestibilité des rations (Jarrige et al 1995, Baumont et al 1997). Dans ces expériences, cependant, les rations ingérées n'étaient pas de composition strictement identique, puisque la quantité de foin dépendait de l'ingestion des animaux. Pour expliquer la différence d'ingestibilité, il est ainsi difficile de dissocier l'effet du mélange, des effets de la taille des brins et/ou de la composition des rations.

En élevage allaitant, l'objectif n'est pas nécessairement de maximiser la croissance hivernale, mais plutôt d'optimiser le rapport «coût de la ration/performance» (Petit 1988) et d'amener les lots à un poids objectif à l'âge choisi. Une «sur-distribution» entraîne une augmentation de la croissance hivernale nécessaire et il est maintenant facilité par la technologie de pesée en continu et l'affichage associé.

b) Effets de la composition du mélange selon l'âge et les besoins des génisses d'élevage

En considérant acquis l'effet décrit précédemment d'augmentation de l'ingestion d'une ration mélangée par rapport à des aliments séparés, la quantité de concentré mise dans le mélange a été réduite systématiquement de $50 \%$ par rapport à la ration témoin au cours d'une deuxième série d'expériences. Cette validation a été réalisée en se basant sur le même protocole que l'expérimentation précédente appliqué à des génisses de 2 ans pendant 94 jours. Ainsi par rapport au lot témoin, la réduction de la quantité totale de concentré consommée a été relativement modeste $(-0,5 \mathrm{~kg}$ par animal et par jour), du fait de l'augmentation de la consommation volontaire de la ration mélangée. Les performances moyennes de croissance des génisses se sont maintenues en considérant l'énergie ingérée, sans modification de la variabilité interindividuelle. En revanche, dans un essai similaire avec des génisses de 1 an, la réduction systématique de $50 \%$ des apports de concentrés s'est révélée trop importante. L'ingestion de la ration mélangée n'a que peu augmenté, et la consommation supplémentaire de fourrages (+ 0,4 kg MS) n'a compensé qu'en partie la réduction du concentré (- 1,5 kg). Les croissances en ont été affectées (tableau 1) en étant même inférieures à celles que l'apport UFL permet de calculer théoriquement (594 vs $750 \mathrm{~g} / \mathrm{j}$ ) (INRA 2007). L'adoption de

Tableau 1. Quantités ingérées et performances zootechniques de génisses d'élevage, selon l'âge et la modalité de distribution de la ration, mélangée ou séparée (Farrié et Renon, non publié).

\begin{tabular}{|r|r|r|r|r|}
\hline & \multicolumn{2}{|c|}{ Génisses 22 mois } & \multicolumn{2}{c|}{ Génisses 10 mois } \\
\hline Poids initial des génisses (kg) & \multicolumn{2}{|c|}{550} & \multicolumn{2}{c|}{380} \\
\hline & $\begin{array}{c}\text { Ration } \\
\text { mélangée }\end{array}$ & $\begin{array}{c}\text { Ration } \\
\text { séparée }\end{array}$ & $\begin{array}{c}\text { Ration } \\
\text { mélangée }\end{array}$ & $\begin{array}{c}\text { Ration } \\
\text { séparée }\end{array}$ \\
\hline Quantités ingérées MS/animal/jour & 11,3 & 10,4 & 7,9 & 8,1 \\
\hline $\begin{array}{r}\text { dont } \\
\text { ensilage herbe }\end{array}$ & 4,9 & 5,2 & 3,2 & 3,8 \\
\hline concentré & 6,0 & 4,3 & 3,5 & 2,5 \\
\hline Apport UFL/animal/jour & 7,8 & 7,6 & 5,8 & 6,8 \\
\hline Concentration énergétique UFL/kg MS & 0,69 & 0,73 & 0,74 & 0,84 \\
\hline Croissance g/j & $605 \pm 169$ & $626 \pm 191$ & 594 & 850 \\
\hline
\end{tabular}


Figure 2. Ration de composition unique distribuée tout l'hiver à des vaches allaitantes : représentation schématique des stratégies comparées.

F : Foin ; Ens. : Ensilage ; Cctré : Concentré.

Stratégie Témoin

«proche des recommandations»
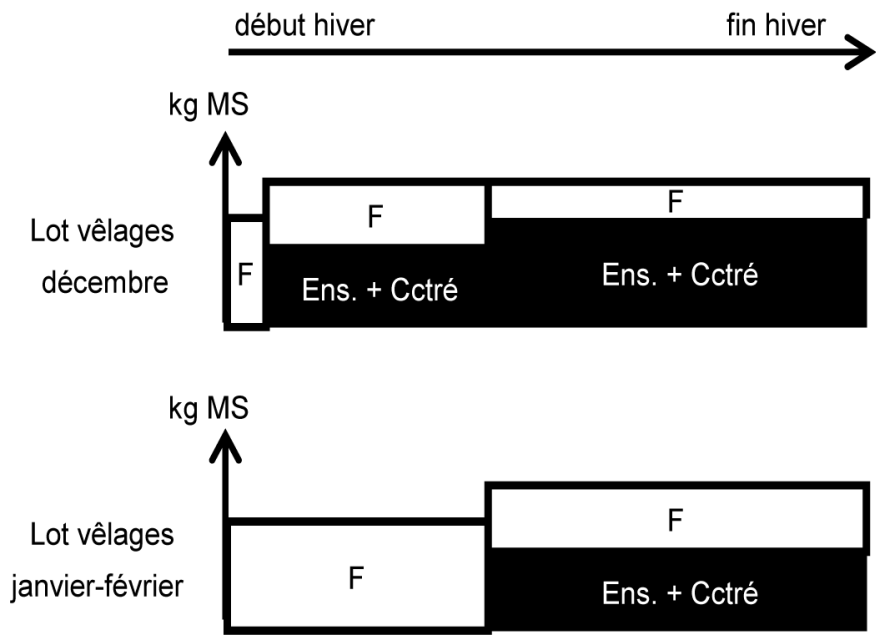

\section{Stratégie}

«simplifiée»
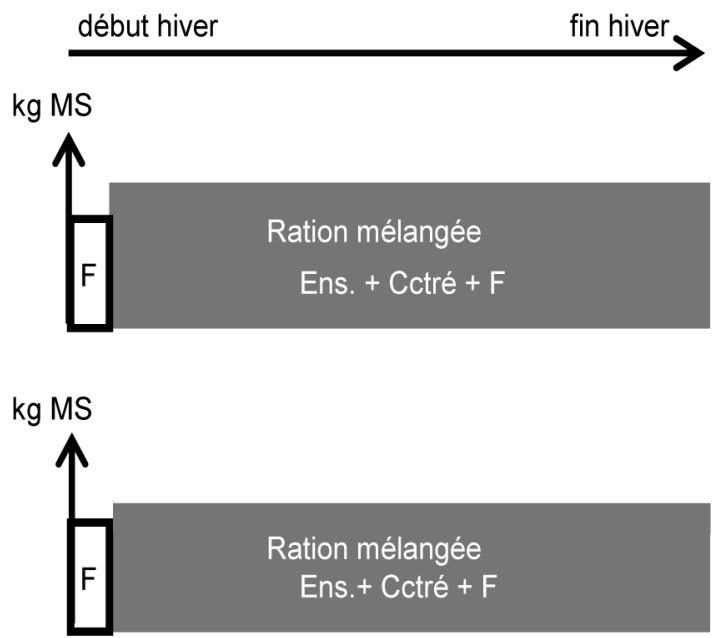

telles pratiques, ration mélangée et réduction de l'apport de concentré, n'est donc pas conseillée avec des objectifs élevés de croissance. On peut au final considérer que l'effet strict du mélange de la ration est double et porte sur son ingestibilité et sa valorisation par l'animal. De ce strict point de vue, l'effet du broyage lié à la mélangeuse semble d'autant plus intéressant que la ration contient initialement moins de concentré, que les objectifs de croissance sont limités et que l'animal est moins exigeant.

Cette série limitée d'essais ne permet pas d'établir une loi de réponse précise de la substitution des aliments concentrés selon l'ingestibilité des rations mélangées. Nous pouvons cependant proposer la démarche suivante dans la pratique. L'estimation de la quantité d'aliments concentrés nécessaires s'effectue par un calcul de ration classique (INRAtion 2008), en considérant une valeur d'encombrement «apparente» des fourrages utilisés. Celle-ci sera alors réduite systématiquement de $10 \%$ par rapport à la valeur UEB théorique. Avec des fourrages de qualité moyenne (foin) à bonne (ensilage d'herbe), cela conduit à une réduction de l'apport de concentré de l'ordre de $1 \mathrm{~kg} / \mathrm{jour}$, quantité indicative qui sera modulée en fonction de la valeur des fourrages utilisés, des catégories d'animaux (âge, race) et des objectifs de croissance.

c) Distribution d'une ration mélangée de composition unique à des lots de vaches allaitantes

L'utilisation de la ration mélangée amène également à s'interroger sur la meilleure stratégie pour l'affouragement de vaches allaitantes. Les lots d'une même étable ont des besoins différents puisque les stades physiologiques des animaux évoluent dans le temps. Pour en tenir compte, deux stratégies sont possibles, l'une qui satisfasse au plus près le besoin de l'animal moyen du lot à une période donnée, l'autre qui laisse chaque animal s'adapter à ses besoins par son comportement alimentaire (vitesse et niveau d'ingestion).

Ces deux stratégies ont été testées sur deux lots de vaches allaitantes, à la ferme de Jalogny, durant trois périodes d'hivernage (2001, 2002, 2003). Le régime alimentaire était toujours composé d'un fourrage humide ensilé (herbe ou maïs selon les hivers), d'un fourrage sec (foin ou de paille) et d'un complément d'aliment concentré. Les modalités de distribution et de répartition des fourrages se différenciaient selon les principes présentés à la figure 2. Dans chaque stratégie les animaux sont regroupés par dates de mise bas similaires (16 décembre ou 16 janvier) en deux cases de 14 animaux.

Pour la stratégie témoin, les rations sont établies de façon à s'approcher au plus près des apports recommandés (INRA 2007) moyens de chaque case. La composition des rations est donc différente d'une case à l'autre, et évolue différemment en cours d'hiver. Pour la stratégie simplifiée, une ration d'étable de composition unique est fabriquée en utilisant chaque jour les mêmes quantités d'ingrédients que pour la stratégie témoin. Elle est distribuée à tous les animaux des deux cases. La répartition des aliments entre les deux cases est contrôlée visuellement de telle sorte que l'évolution en cours de journée de la quantité de fourrages disponible à l'auge soit similaire.

Les deux stratégies conduisent à distribuer la même quantité globale de fourrages et de concentrés à l'échelle de l'hiver, soit 975, 1053 et 1075 UFL/ vache pour les trois années d'expérimentation ayant duré respectivement 96, 99 et 106 jours. Mais la répartition dans le temps et entre lots est différente. Un bilan plus fin montre que, par rapport au témoin, les vaches des lots simplifiés vêlant en décembre tendent à être légèrement sous-alimentées (- $0,3 \mathrm{UFL} / \mathrm{j}$ soit - $3,1 \%$ ), alors que c'est le contraire pour les animaux des lots vêlant en janvier $(+0,5 \mathrm{UFL} / \mathrm{j}$ soit $+8 \%$ ). Cette différence apparaît surtout durant la première moitié de l'hiver, période pendant laquelle l'hétérogénéité des besoins des animaux est la plus grande (Farrié et al 2004). L'effet de la simplification se traduit donc par des différences dans l'évolution du poids et de la Note d'Etat Corporel (NEC) des vaches (tableau 2).

Bien que les évolutions soient contrastées entre les trois années, elles sont généralement en défaveur de la stratégie simplifiée. Cet effet est surtout toujours perceptible pour les mères qui vêlent le plus tôt. Mais aucune répercussion sur la vitalité des veaux à la naissance ni sur leur croissance ultérieure n'a pu être mise en évidence. Enfin, aucun arrière effet supplémentaire n'a été observé, que ce soit sur la croissance ultérieure des veaux après la phase hivernale, ou sur la réussite du 
Tableau 2. Evolution moyenne du poids vif (corrigé des produits de la gestation) et de la Note d'Etat Corporel (NEC) de vaches Charolaises recevant une ration composée d'ensilage et de foin séparés, ou mélangés, et selon la période de vêlage.

Moyenne de 3 années d'observations pour une période de 100 j par hiver.

\begin{tabular}{|l|c|c|c|}
\hline Vêlages «décembre» & $\begin{array}{c}\text { Ration } \\
\text { séparée }\end{array}$ & $\begin{array}{c}\text { Ration } \\
\text { mélangée }\end{array}$ & $\begin{array}{c}\text { Effet du type } \\
\text { de ration }\end{array}$ \\
\hline Poids final - Poids intial $(\mathrm{kg})$ & +1 & -10 & $\mathrm{p}<0,1$ \\
$+/$ - écart-type & \pm 30 & \pm 35 & \\
\hline NEC finale - NEC initiale & $-0,34$ & $-0,51$ & $\mathrm{p}<0,05$ \\
$+/$ - écart-type & $\pm 0,39$ & $\pm 0,51$ & \\
\hline Vêlages ajanvier-février» & & & \\
\hline Poids final - Poids intial (kg) & -19 & -12 & $\mathrm{NS}$ \\
$+/$ - écart-type & \pm 38 & \pm 36 & \\
\hline NEC finale - NEC initiale & $-0,51$ & $-0,44$ & $\mathrm{NS}$ \\
$+/$ écart-type & $\pm 0,39$ & $\pm 0,51$ & \\
\hline
\end{tabular}

retour à la reproduction des mères. La modification des pratiques d'alimentation s'est donc traduite par une réponse des vaches de faible ampleur, uniquement observée sur leur état corporel. Ce recours aux réserves corporelles traduit au final une bonne adaptation des vaches à cette simplification de pratique.

Au-delà de la modification du travail et de sa pénibilité discutées par ailleurs (Hostiou et Fagon 2012), les conséquences de l'utilisation d'une ration mélangée, sur le comportement et les performances des animaux à besoins modérés, sont nombreuses, et ne peuvent toutes être présentées dans cet article. Nous retenons cependant l'effet favorable du broyage mécanique partiel sur l'ingestibilité de ces rations riches en fourrages grossiers. Nous pouvons aussi souligner l'économie associée d'aliment concentré si les objectifs de croissance le permettent. Les conséquences zootechniques demeurent modestes. En revanche, le risque de «sur-distribution» de cette ration de bonne valeur énergétique existe. Le contrôle régulier de la distribution doit permettre d'éviter un gaspillage toujours possible par des animaux à faibles besoins.

\section{2 / Simplifier la distribution des aliments concentrés}

La pénibilité de la distribution des aliments concentrés est moindre que la distribution de fourrages. Mais elle peut être fastidieuse dans les cas où ces aliments correspondent à la plus grande (Normand et al 2003).
Pourtant, Reyne et Garambois (1977), ont observé que la distribution séparée de la céréale et du protéagineux n'avait pas entraîné de surconsommation laissant entrevoir une possibilité de simplification importante aussi bien dans le cas d'une distribution manuelle que mécanisée. A partir de ce constat, un ensemble de 10 essais a été conduit entre 2001 et 2003 en stations expérimentales sur des agneaux de différents types génétiques (Vendéen, croisés Charollais) avec deux protéagineux, le pois et le lupin (Normand et al 2003). La distribution séparée des différents constituants de la ration a été comparée à une distribution mélangée. Dans un des essais, les agneaux ont été conduits en case individuelle pour évaluer la variabilité individuelle des choix alimentaires.

A l'exception d'un essai, la distribution dans des trémies séparées des constituants de la ration n'a pas engendré de surconsommation de l'un ou de l'autre des aliments sur l'ensemble de la phase de finition. Ils ont été consommés soit dans des proportions proches des recommandations, pour un tiers des essais portant sur le pois et la moitié des essais portant sur le lupin, soit avec un taux de protéagineux un peu plus élevé (figure 3).

Cependant une très forte variabilité de comportement alimentaire a été observée entre individus, certains présentant une préférence marquée pour le pois, d'autres pour la céréale ; cette préférence pouvant également fortement varier quotidiennement (figure 4). Mais, en prenant la précaution que les différents constituants soient bien consommés dans leur totalité, la distribution séparée, en comparaison de la distribution

Figure 3. Incidence d'une distribution séparée de la céréale et du protéagineux sur la proportion de protéagineux consommée dans la ration par des agneaux en finition

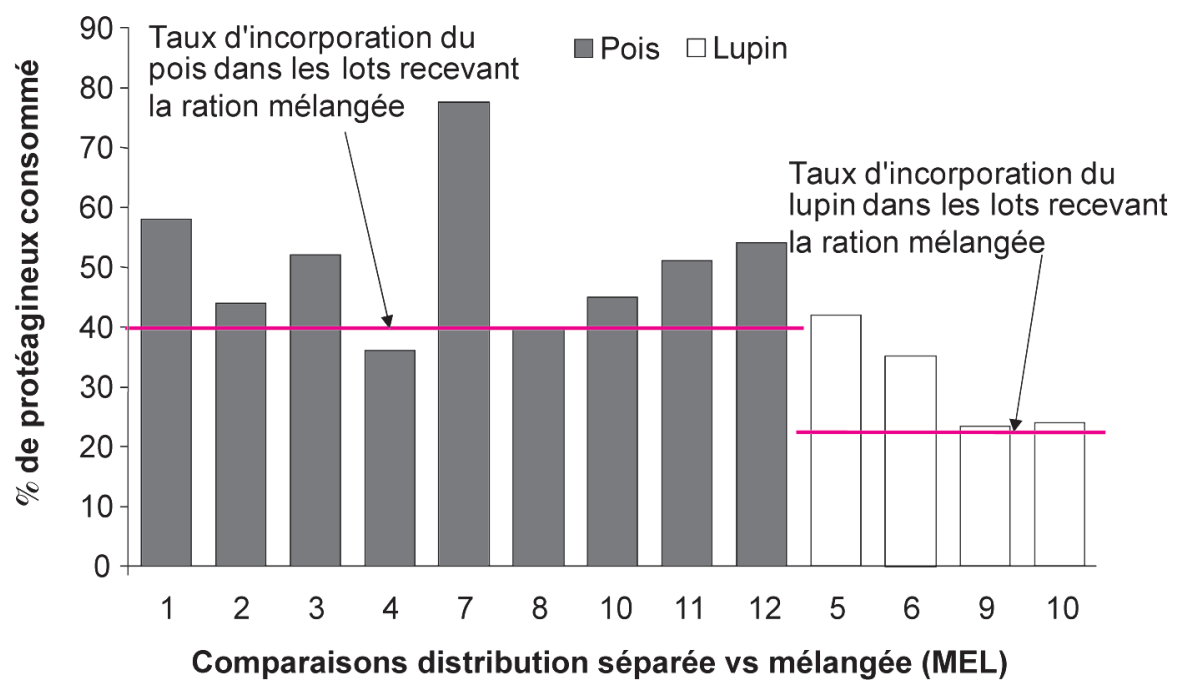


Figure 4. Variabilité individuelle de la proportion de pois consommé quotidiennement par des agneaux en finition lorsque cet aliment est distribué séparément d'une céréale (Normand et al 2003).

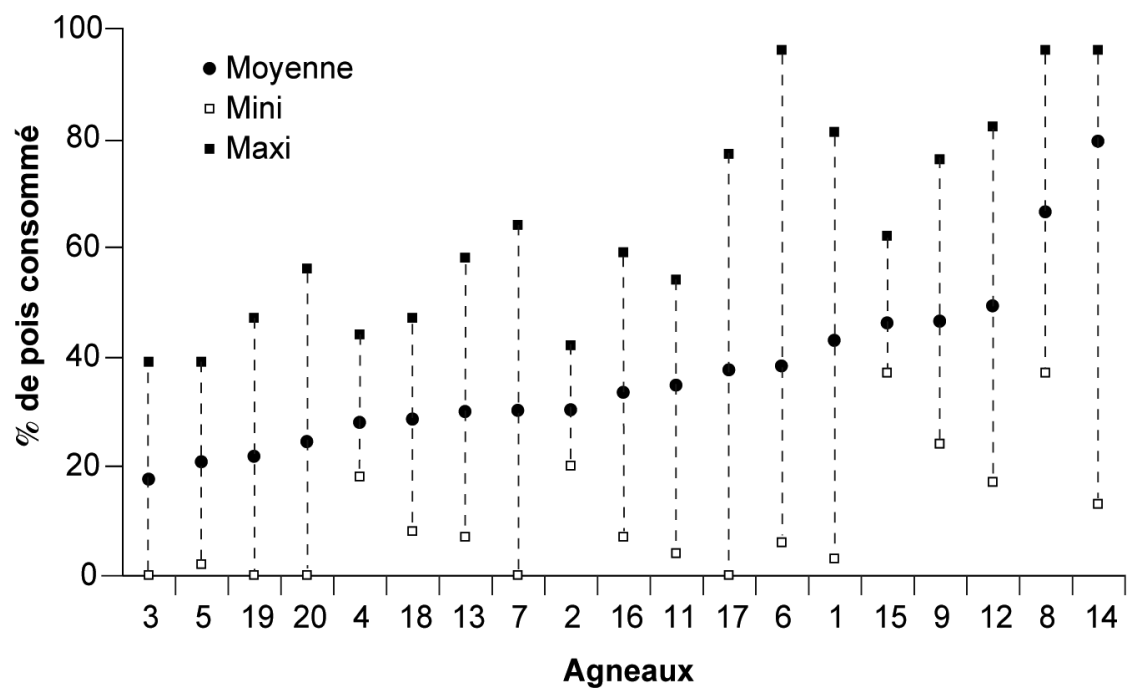

mélangée, ne s'est traduite ni par des croissances différentes ni par une plus grande hétérogénéité des performances. Aucune différence n'a été observée sur le taux de mortalité ni sur la fréquence des troubles digestifs.

Enfin, il faut noter que sur la base des teneurs en MAT du pois comme du lupin (INRA 2007), les taux de protéagineux de la ration sont en moyenne, de 50 et $31 \%$ respectivement, et sont ainsi conformes aux recommandations.

Ces résultats confirment la capacité des agneaux à s'autoréguler et laissent entrevoir des solutions simples à mettre en œuvre pour faciliter l'utilisation d'aliments fermiers et concilier les

recherches conjointes d'une plus grande autonomie alimentaire et de moindres contraintes de travail.

b) Limiter la consommation de concentré en limitant le temps d'accès

La simplification de l'alimentation des agneaux en finition peut passer par des solutions peu contraignantes pour aider au rationnement du concentré. La pratique habituelle d'un apport quotidien de concentré en quantité individuelle déterminée, a pour objectif de mieux maîtriser leur état d'engraissement ainsi que d'améliorer la qualité des gras de couverture en couleur et tenue (Normand et Brouard 2001). Des travaux réalisés à la fin des années 1990
Figure 5. Incidence du temps d'accès à l'auge sur la réduction de la consommation journalière de concentré par des agneaux (observations dans trois sites expérimentaux).

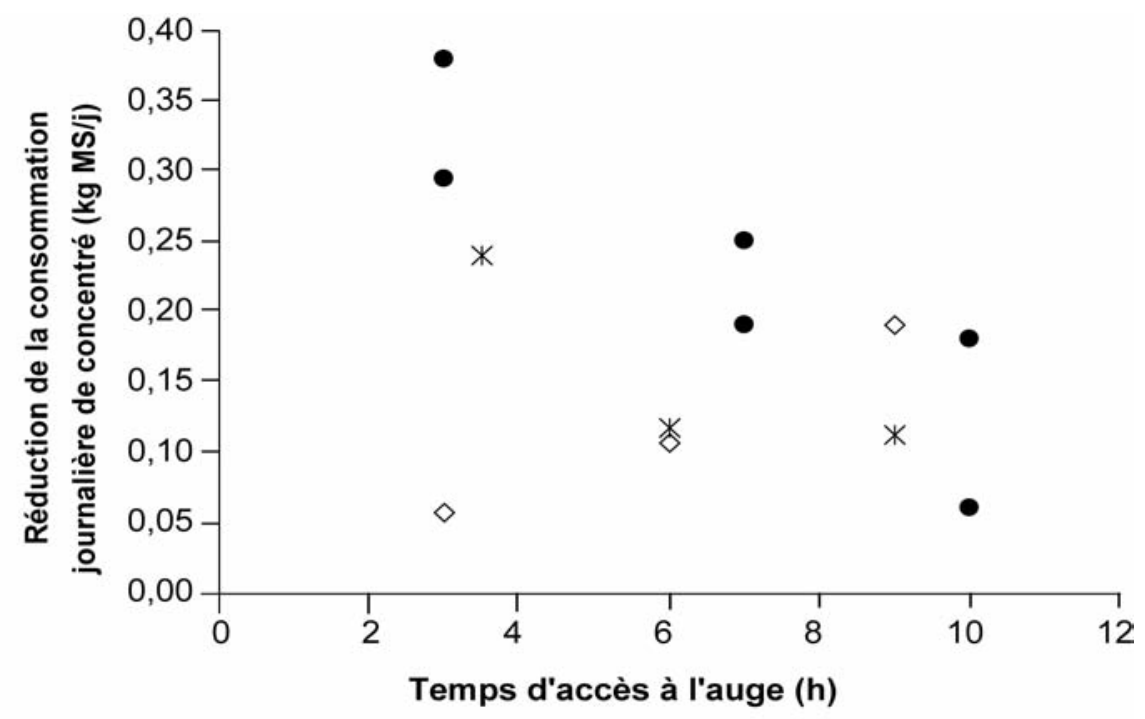

- Le Mourier $\diamond$ Glane $*$ Mauron
(Normand et al 2003) pendant trois années consécutives ont montré qu'à l'exception d'un type génétique (Lacaune $\mathrm{x}$ Charollais), la restriction du temps d'accès des agneaux à la trémie s'est traduite par une baisse de la consommation quotidienne (figure 5). Avec un temps d'accès quotidien limité à $3 \mathrm{~h}$ consécutives, le taux de rationnement dépassait $20 \%$ pour les races à viande (par exemple, Mouton Vendéen, ou Rouge de l'Ouest). Conjointement la qualité des gras de couverture s'est améliorée, en lien fort avec la réduction de l'énergie quotidiennement ingérée $(\mathrm{r}=-0,58 ; \mathrm{n}=12 ; \mathrm{p}<0,05)$.

Ces approches originales de la distribution d'aliment concentré à des agneaux montrent clairement que les simplifications de certaines pratiques d'alimentation peuvent être à la fois intéressantes pour l'éleveur et répondre à des enjeux de qualité pour la filière.

\section{2 / Simplifier en modifiant le rythme de distribution des aliments}

\section{1 / Moduler le rythme de dis- tribution au cours de la semaine}

Pour l'ensemble des essais réalisés et répertoriés dans le tableau 3, l'objectif était de simplifier le travail d'affouragement. Plusieurs modalités ont été testées: la réduction de la fréquence journalière de distribution d'une même ration, la mise en place d'une distribution alternée de fourrages différents pour éviter des travaux pénibles le week-end, enfin la mise en place d'une ration «hebdomadaire» au lieu de quotidienne, rendue possible par la forme de récolte et de stockage des fourrages. Ces pratiques simplifiées, testées sur une large gamme de types de bovins laitiers et allaitants, consistent à espacer les distributions sans modifier profondément les équipements et installations existantes. Elles mobilisent toutes à la fois la plasticité de l'ingestion et de la capacité digestive des ruminants.

a) Conséquences sur l'ingestion et les performances des génisses d'élevage ou des vaches en production

Ces observations ont été principalement réalisées en station expérimentale sur des bovins dont les niveaux de production sont limités et dont les phases de récupération potentielle par la suite au pâturage sont maîtrisées. Les indicateurs de performances utilisés sont 1) les quantités ingérées par rapport à un témoin alimenté sur un rythme quotidien, 2) les modifications de comportement d'ingestion et 3) la production, 
Tableau 3. Impacts des modifications du rythme de la distribution de la ration sur les performances de lots de bovins allaitants et laitiers.

\begin{tabular}{|c|c|c|c|c|c|c|c|c|}
\hline \multirow{2}{*}{$\begin{array}{l}\text { Fréquence } \\
\text { et mode de } \\
\text { distribution }\end{array}$} & \multirow{2}{*}{$\begin{array}{c}\text { Type } \\
\text { d'animal }\end{array}$} & \multirow{2}{*}{ Rations } & \multirow{2}{*}{$\begin{array}{l}\text { Observations } \\
\text { ou nombre de } \\
\text { répétitions }\end{array}$} & \multicolumn{4}{|c|}{$\begin{array}{l}\text { Impacts sur les performances } \\
\text { par rapport à un témoin } \\
\text { (rationnement une fois par jour) }\end{array}$} & \multirow[t]{2}{*}{ Références } \\
\hline & & & & Ingestion & $\begin{array}{l}\text { Comportement } \\
\text { alimentaire }\end{array}$ & $\begin{array}{l}\text { Gain ou perte } \\
\text { de poids }\end{array}$ & $\begin{array}{l}\text { Carcasse } \\
\text { ou } \\
\text { lait produit }\end{array}$ & \\
\hline $\begin{array}{l}2 \text { repas/jour } \\
\text { vs } \\
1 \text { repas/jour }\end{array}$ & \multirow[t]{2}{*}{$\begin{array}{l}\text { Bouvillons } \\
\text { engrais }\end{array}$} & \multirow{2}{*}{$\begin{array}{c}\text { Mélange : } \\
\text { Foin }(10 \%) \\
+ \text { Maïs grain }(65 \%) \\
\text { + Tourteau Soja }(5 \%) \\
+ \text { Minéraux. }\end{array}$} & 2 & NS & \multirow{2}{*}{$\begin{array}{c}\text { Fortes } \\
\text { variations } \\
\text { individuelles }\end{array}$} & $\begin{array}{l}\text { Identique ou } \\
\text { très peu } \\
\text { différent }\end{array}$ & NS & \multirow{2}{*}{$\begin{array}{l}\text { Soto Navarro } \\
\text { et al (2000) } \\
\text { Schutz et al } \\
(2011)\end{array}$} \\
\hline 3 jour vs 1 jour & & & 2 & $\begin{array}{c}\text { NS ou } \\
p<0,04\end{array}$ & & $p<0,03$ & $p<0,01$ & \\
\hline \multirow{3}{*}{$\begin{array}{l}3 \text { fois } \\
\text { par semaine } \\
\text { (lundi, } \\
\text { mercredi } \\
\text { vendredi) }\end{array}$} & $\begin{array}{c}\text { Génisses } \\
1 \text { an }\end{array}$ & $\begin{array}{l}\text { Ens. maīs }(5 \mathrm{~kg})+ \\
\text { Tourteau Soja }(0,7 \mathrm{~kg}) \\
\text { + Paille ad lib. }\end{array}$ & 3 & Identique & 1 & Identique & 1 & \multirow{3}{*}{$\begin{array}{l}\text { Bastien et al } \\
\text { (2009) }\end{array}$} \\
\hline & $\begin{array}{l}\text { Vaches } \\
\text { allaitantes }\end{array}$ & $\begin{array}{l}\text { Ens. d'herbe } \\
\text { (6 à } 8,5 \mathrm{~kg}) \\
+ \text { Foin } a d \mathrm{lib} . \\
+ \text { Blé (env. } 1 \mathrm{~kg})\end{array}$ & 3 & $\begin{array}{c}\text { de }+0,5 \mathrm{a} \\
+1,5 \mathrm{~kg} \\
(2,5 \%)\end{array}$ & 1 & $\begin{array}{l}+20 \mathrm{~kg} \text { sur } \\
\text { l'hiver (NS) } \\
\text { primipare et } \\
\text { multipare }\end{array}$ & $\begin{array}{l}\text { Veaux : } \\
\text { GMQ } \\
\text { identiques }\end{array}$ & \\
\hline & Taurillons & $\begin{array}{l}\text { Ens. maîs (ad lib.) } \\
+ \text { Concentre }(3,3 \mathrm{~kg}) \\
\text { + Paille ad lib. }\end{array}$ & 4 & $\begin{array}{c}\text { Hiver }+3,5 \% \\
\text { Eté : - } 2 \%\end{array}$ & $\begin{array}{l}\text { Repas plus } \\
\text { réguliers } \\
\text { et plus brefs }\end{array}$ & $\begin{array}{c}-35 \mathrm{a} \\
-110 \mathrm{~g} / \mathrm{jour} \\
(\mathrm{NS})\end{array}$ & $\begin{array}{l}-15 \mathrm{~kg} \\
\text { carcasse } \\
p<0,01\end{array}$ & \\
\hline $\begin{array}{l}2 \text { fois } \\
\text { par semaine }\end{array}$ & $\begin{array}{l}\text { Vaches } \\
\text { laitières }\end{array}$ & $\begin{array}{c}\text { Ens. maïs } \\
+ \text { Concentré } \\
+ \text { Stabilisateur ensilage }\end{array}$ & 1 & Identique & 1 & 1 & Identique & $\begin{array}{l}\text { Bonnefoy et } \\
\text { a/ }(2010)\end{array}$ \\
\hline \multirow{3}{*}{$\begin{array}{l}\text { Distribution } \\
\text { alternée } \\
\text { de fourrages } \\
\text { differents } \\
\text { (pas d'ensilage } \\
\text { d'herbe } \\
\text { le week-end) }\end{array}$} & $\begin{array}{c}\text { Génisses } \\
1 \text { an }\end{array}$ & $\begin{array}{c}\text { Ens. herbe/foin }(33 \%) \\
\text { ou } \\
\text { Ens. herbe/ens. maïs } \\
(45 \%)\end{array}$ & 5 & \multirow{3}{*}{$\begin{array}{l}\text { Identique par } \\
\text { construction }\end{array}$} & 1 & $\begin{array}{c}-130 \mathrm{~g} / \mathrm{jour} \\
p<0,01\end{array}$ & 1 & \multirow{3}{*}{$\begin{array}{l}\text { Grenet ef al } \\
(1997)\end{array}$} \\
\hline & $\begin{array}{c}\text { Génisses } \\
2 \text { ans }\end{array}$ & $\begin{array}{c}\text { Ens. herbe/paille }(20 \%) \\
\text { ou } \\
\text { Ens. herbe/foin }(33 \%)\end{array}$ & 6 & & 1 & $\begin{array}{c}-70 \text { g/jour } \\
p<0,05\end{array}$ & 1 & \\
\hline & $\begin{array}{c}\text { Vaches } \\
\text { allaitantes }\end{array}$ & Enrubanné/foin $(25 \%)$ & 4 & & 1 & $-7 \mathrm{~kg} \mathrm{NS}$ & $\begin{array}{l}\text { Veaux: } \\
-5 \mathrm{~kg}\end{array}$ & \\
\hline $\begin{array}{l}\text { Hebdomadaire } \\
\text { vs quotidienne } \\
\text { du foin }\end{array}$ & $\begin{array}{l}\text { Vaches en } \\
\text { gestation } \\
\text { puis } \\
\text { allaitantes }\end{array}$ & $\begin{array}{c}\text { Foin }(7 \times 9 \mathrm{~kg} \\
\text { ou } 63 \mathrm{~kg}) \\
\text { Paille ad lib }\end{array}$ & 2 & $\begin{array}{l}\text { Paille : - } 0,7 \\
\text { kg }\end{array}$ & $\begin{array}{l}\text { Foin totalement } \\
\text { ingéré } \\
\text { en } 4 \text { jours }\end{array}$ & $\begin{array}{l}-14 \mathrm{~kg} \\
p<0,05\end{array}$ & $\begin{array}{c}\text { Lait } \\
-0,3 \mathrm{~kg} / \mathrm{jour} \\
\text { Veaux } \\
-65 \text { g/jour } \\
\text { NS }\end{array}$ & $\begin{array}{l}\text { Lassalas et a! } \\
(1997)\end{array}$ \\
\hline
\end{tabular}

le gain de poids ou la production de lait dans les quelques cas où il s'agissait de vaches laitières.

Pour des génisses comme pour des vaches allaitantes, le temps gagné en ne distribuant la ration d'ensilage que trois fois par semaine s'élève à $30 \%$ environ (Bastien et al 2009). Cette estimation a été faite en conditions expérimentales et sans modifier la quantité totale hebdomadaire donnée aux animaux. Les performances n'ont pas été dégradées et les gains de poids des génisses de 1 an sur 105 jours sont restés voisins de $750 \mathrm{~g} / \mathrm{j}$. Ce même résultat s'est retrouvé sur les lots de vaches allaitantes qui ont perdu un peu moins de poids et d'état que les lots témoins.

Ce n'est pas le cas des essais de distributions alternées de fourrages de qualités différentes : foin grossier vs ensilage de maïs ou d'herbe (Grenet et al 1997). Le rythme simplifié consistait à suppri- mer les distributions d'ensilage pendant les week-ends qui peuvent sembler fastidieuses et coûteuses. Pendant cinq jours de la semaine les lots d'animaux recevaient les aliments humides et les deux jours suivant ils recevaient le reste de la ration correspondant aux fourrages grossiers (foin ou paille). Cette pratique n'a pas entraîné de problèmes sanitaires, mais a réduit d'autant plus les gains de poids des génisses qu'elles étaient jeunes, respectivement de - 130 $\mathrm{g} / \mathrm{j}$ et de $-80 \mathrm{~g} / \mathrm{j}$ à 1 an et à 2 ans.

Ces deux séries d'expériences conduisent à des réponses différentes des animaux qui pourraient s'expliquer par les possibilités d'adaptation et de compensation que le dispositif expérimental offrait aux animaux. Dans la première série d'expériences, seul l'ensilage était distribué selon le rythme de trois distributions par semaine et un fourrage supplémentaire de qualité médiocre était toujours disponible à volonté. Les génisses ont sans doute pu compenser en qualité et en quantité et équilibrer leur ration dans la durée. Dans la seconde série d'expériences, les animaux recevaient des quantités d'aliments strictement identiques distribuées au «cornadis» dans les deux traitements. Si la distribution alternée se traduit par une moindre utilisation digestive des aliments de la ration, sans possibilité de compensation quantitative, la performance animale est très logiquement réduite.

Cependant ces deux essais (Grenet et al 1997, Bastien et al 2009) (tableau 3) montrent que les vaches adultes sont moins sensibles que les génisses à une modification du rythme de distribution de la ration. Elles ne perdent que peu de poids, et de manière non significative par rapport au lot témoin. Les plus jeunes génisses subissent davantage les changements nutritionnels réguliers mais brutaux et seraient donc moins 
flexibles à l'échelle des ces quelques mois d'observation. A l'échelle de leur carrière on peut penser qu'elles disposeraient de possibilités de compensation, au moins pendant les périodes de pâturage (Hoch et al 2003). L'ensemble de ces résultats justifient pleinement de promouvoir les pratiques simplifiées pour tous ces animaux tout en étant cependant plus vigilant pour les plus jeunes.

En élevage, les rations sont habituellement distribuées quotidiennement. Elles sont souvent composées de deux fourrages de valeurs très différentes (foin et paille par exemple), récoltés en grosses balles et distribués l'un et l'autre chaque jour dans des râteliers, ce qui augmente le temps de travail. Le meilleur foin est distribué en quantité limitée. Ne l'offrir qu'une fois, au début de la semaine permettrait de réduire la fréquence de la distribution et constituerait un gain de temps substantiel. Les conséquences d'un tel changement ont été analysées dans deux essais comparables répétés au site des Razats de l'Unité Expérimentale INRA des Monts d'Auvergne (Lassalas et al 1997). Ainsi, un lot expérimental de vaches en fin de gestation puis en lactation, a reçu une ration selon un rythme hebdomadaire, soit $9 \mathrm{~kg} \times 7$ jours $=63 \mathrm{~kg}$ de foin de prairie permanente par tête $(60 \%$ de digestibilité), distribuée chaque lundi et de la paille à volonté. Les vaches témoins ont reçu chaque jour de la semaine la même ration $(9 \mathrm{~kg}$ de foin par animal et paille à volonté). Les ingestions de foin et de paille ont été mesurées quotidiennement dans les deux lots (figure 6). Les périodes expérimentales ont duré en moyenne onze semaines, les vêlages étant répartis de la première à la cinquième semaine d'expérimentation. Tous les jours, les refus de tous les fourrages dans l'auge ont été mesurés.

La réponse adaptative comportementale porte sur la dynamique de l'évolution hebdomadaire des quantités ingérées et sur la permanence de cette adaptation pendant l'expérimentation. En début de semaine les vaches ingèrent uniquement du foin, puis au fur et à mesure que le stock diminue la consommation de paille augmente : à partir du cinquième jour, elles ne disposent plus que de paille. L'énergie ingérée par vache diminue donc de 13 à 3 UFL entre le lundi et le vendredi, et simultanément l'encombrement calculé de la ration passe de 21 à 10 UEB avant de s'élever à nouveau le dimanche à 16 UEB. Les choix des animaux sont très significatifs : tant que du bon fourrage est disponible les vaches l'ingèrent. Elles ne se reportent sur la paille qu'au bout du troisième jour (figure 6).

Figure 6. Effet de la distribution du foin une fois par semaine (ration simplifiée) sur les quantités ingérées journalières de foin et de paille exprimées en UEB, et sur l'énergie ingérée exprimée en UFL (Lassalas et al 1997).

Le lot témoin reçoit une ration journalière fixe de foin.

Moyenne des semaines expérimentales 9 à 11 pour des vaches allaitantes Salers et Charolaises (poids vif $=680 \mathrm{~kg}$; Note d'état $=2,3 / 5$; Production de lait $=8,4 \mathrm{~kg}$ ).

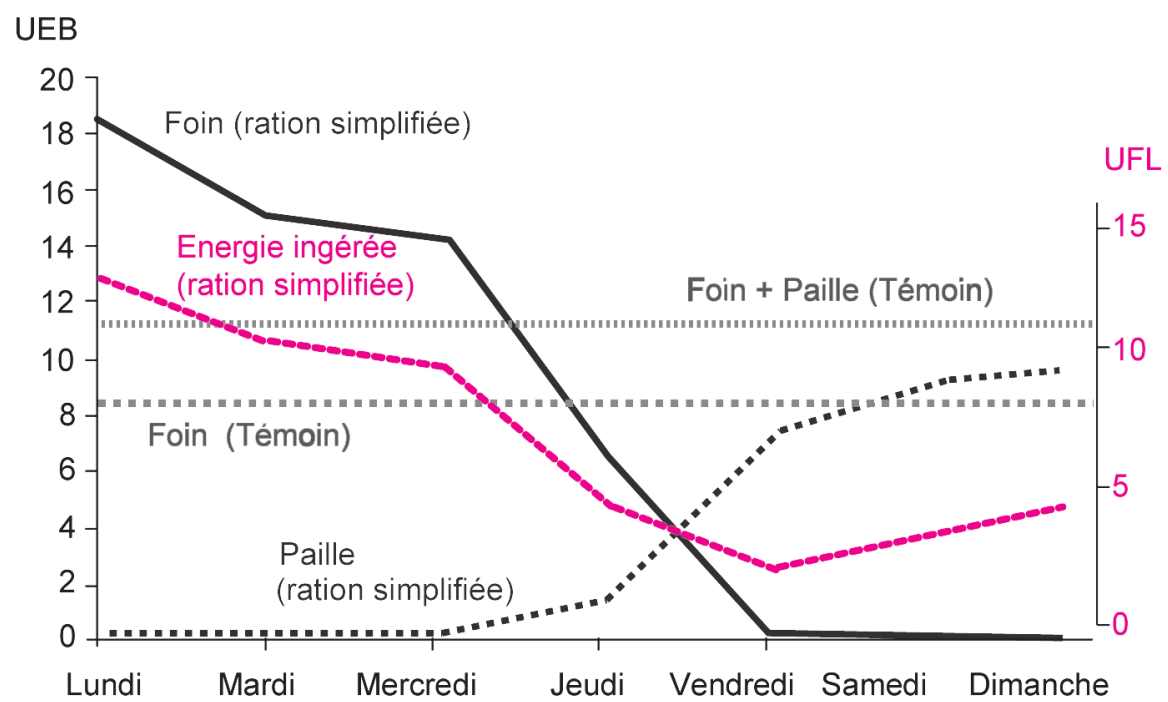

Ce comportement peut s'expliquer en partie par l'importance de l'écart de valeurs d'encombrement entre les deux fourrages, mais dans ce cas le niveau d'ingestion de foin en début de semaine ne devrait pas dépasser la capacité d'ingestion 16 UEB.

Ainsi dans les deux essais, le foin a toujours été ingéré en totalité quel que soit le lot. Du fait de l'évolution du stade physiologique, l'ingestion de paille a augmenté progressivement au cours de l'expérience passant de 1,8 à $3,9 \mathrm{~kg} \mathrm{MS} / \mathrm{j}$. La distribution hebdomadaire a entraîné une baisse significative des quantités moyennes ingérées de paille, qui reste cependant modeste $(-0,7 \mathrm{~kg} / \mathrm{j})$ mais répétée chaque année. Cet écart entre les deux lots a tendance plutôt à se réduire au cours de la période expérimentale. Au total il correspond à une faible différence d'apports énergétiques journaliers calculés $(0,3$ $\mathrm{UFL} / \mathrm{j}$ ) soit une différence totale de 21 UFL entre lots. Cette différence se répercute sur les pertes de poids des vaches puisque, les deux années, les animaux du lot simplifié ont perdu $14 \mathrm{~kg}$ de plus que les témoins. Il en est de même de la perte d'état corporel (- 0,2 point d'écart lors de l'expérience équivalent à 40 UFL environ). En revanche, les productions laitières $(8,5$ et 8,3 $\mathrm{kg} / \mathrm{j})$ et les croissances des veaux (911 $\mathrm{g} / \mathrm{j}$ et $845 \mathrm{~g} / \mathrm{j}$ ) n'ont pas été différentes. Le mode de distribution simplifié des fourrages a induit la même réponse que celle observée en cas de sous alimentation énergétique modérée (Petit et Agabriel 1993). Les vaches ont mobili- sé davantage leurs réserves corporelles mais n'ont pas produit moins.

Ces résultats illustrent aussi que l'encombrement, principal facteur du contrôle quantitatif de l'ingestion des fourrages, peut être modulé par les caractéristiques des aliments de la ration au travers des interactions qui trouvent leur origine dans la perception que les animaux ont des aliments (Ginane et al 2009). Ils montrent que la composante hédonique des choix alimentaires va au-delà des seuls facteurs digestifs et nutritionnels avec pour conséquence la moindre valorisation de l'ensemble de l'offre fourragère (Favreau et al 2010).

$\mathrm{Au}$ final pourtant, une moindre valorisation de la ration hivernale distribuée sur un rythme hebdomadaire, n'a aucun effet à l'échelle du cycle de production car si la saison de pâturage le permet, la récupération des modestes effets est tout à fait possible.

b) Conséquences sur les niveaux d'ingestion et les performances de jeunes bovins à l'engrais

Avec des rations riches en aliment concentré, les durées totales journalières d'ingestion de jeunes taurillons sont assez réduites et ne dépassent guère 2 heures (135 min pour des taurillons Blonds d'Aquitaine alimentés avec une ration d'ensilage de maïs) (Mialon et al 2008). Mais les repas sont nombreux (de 12 à 14) et la vitesse d'ingestion soutenue (de 60 à $170 \mathrm{~g} / \mathrm{min}$ ). Dans le cas d'une seule distribution journalière, 
les taurillons effectuent un repas principal le matin dans les heures qui suivent la distribution et un autre repas important le soir. Dans le cas des rations d'engraissement distribuées tous les trois jours (Bastien et al 2009), l'organisation des repas se déstructure le deuxième jour. Les animaux réalisent alors plusieurs petites séquences d'ingestion ( 9 en moyenne) de durées voisines. Bien que $60 \%$ de la MS soit ingérée le premier jour, les repas sont plus réguliers le jour suivant et les animaux ruminent davantage. Les conditions de bien-être de ces taurillons ne se seraient donc a priori pas dégradées.

Les animaux peuvent ainsi moduler le temps consacré à l'alimentation dans la journée en fonction de la quantité d'aliment disponible dans l'auge et reporter des séquences d'alimentation. Ces adaptations sont moins précisément quantifiées dans la littérature car leur étude nécessite de lourds dispositifs d'observation et les processus mis en œuvre par les animaux sont très variés. Les essais de Schwartzkopf et al (2004) avec des bouvillons à l'engrais en «feed-lot», étaient surtout destinés à montrer l'intérêt de réaliser plus d'une distribution de la ration par jour, afin d'accroître l'ingestion et l'efficacité alimentaire. Néanmoins, ces résultats sont intéressants, parce que comparables à ceux observés en situation de simplification des modalités de distribution. Ils montrent en effet que deux à trois distributions par jour n'améliorent qu'à la marge les performances de croissance sans modifier le ratio d'efficacité alimentaire (Soto-Navarro et al 2000 , Schutz et al 2011) (tableau 3). Les variations individuelles du comportement alimentaire d'un jour à l'autre sont toujours très importantes. De façon plutôt inattendue, ce sont les animaux les plus performants qui seraient ceux dont les fluctuations quotidiennes d'ingestion sont les plus prononcées (Schwartzkopf et al 2011). Mais lorsque la variation des apports quotidiens d'une même ration est l'objet de l'expérimentation, les fluctuations quotidiennes étant donc créées par le dispositif expérimental, celle-ci entraîne une réduction des performances à mêmes quantités ingérées (Soto Navarro et al 2000). Dans cette série d'expériences les auteurs ont également montré qu'une même variation de $\pm 10 \%$ des apports alimentaires est moins perturbante lorsqu'elle est appliquée sur des séquences d'une semaine que lorsqu'elle est appliquée de jour en jour $( \pm 10 \%$ chaque jour). Dans le cas des modulations hebdomadaires, les performances de croissance varient moins, soulignant ainsi la bonne adaptation des bouvillons à cette pratique. Enfin cette adaptation à des fluctuations d'apports a été d'autant plus aisée que les animaux étaient plus âgés et que l'engraissement plus avancé.

\section{c) Risques associés liés à l'évolution} de la qualité de la ration dans l'auge

Dans les expériences déjà citées de distributions simplifiées de rations d'ensilage de maïs à des taurillons Charolais à l'engrais (Bastien et al 2009) (tableau 3), d'autres facteurs, liés à la qualité de la ration, modulent les réponses et les performances. La pénalisation de la production par la pratique de simplification est d'autant plus forte que la température extérieure est plus élevée. Ainsi par rapport aux lots témoins, des taurillons nés en automne qui n'étaient affouragés que trois fois par semaine ont ingéré davantage pendant la période hivernale de finition $(+3,5 \%)$ que pendant la période d'été (- 2\%). Cet effet est particulièrement pénalisant lorsque l'éleveur souhaite finir ses jeunes mâles en juillet. A cette saison, aussi bien les poids que les rendements en carcasse sont légèrement inférieurs. La baisse de l'ingestibilité de la ration observée en été serait liée à une reprise des fermentations de l'ensilage de maïs dans l'auge lorsque la température s'élève.

Pour mieux comprendre cet effet, un autre essai a été réalisé sur des vaches laitières en fin de lactation. Ce sont en effet des animaux encore plus sensibles à la qualité de la ration (Bonnefoy et al 2010). Pendant 8 semaines, des vaches Holstein ont été alimentées avec une ration complète à base d'ensilage de maïs $(70 \%$ de la MS) et d'aliments concentrés, distribuée 3 fois par semaine ou bien tous les jours. Un lot supplémentaire a reçu la même ration complète d'ensilage et de concentré, à laquelle un additif limitant les reprises de fermentation a été ajouté dans l'auge. Quantités ingérées et performances laitières (production de lait, taux protéiques et matières grasses) ont été identiques entre les trois lots ce qui souligne la capacité des animaux fort producteurs à s'adapter à cette simplification de l'affouragement. En revanche, les quantités de spores butyriques du lait ont été accrues pour le lot qui recevait en affouragement simplifié la ration non traitée dans l'auge. Les mesures de température des rations dans les mangeoires ont confirmé les reprises de fermentations, et leur limitation par l'additif.

Les conséquences de la température ambiante (de 10 à $30^{\circ} \mathrm{C}$ ) et celles de la proportion de concentré ( 0 à $40 \%$ ) sur la qualité sanitaire des rations complètes à base d'ensilage ont pu être analysées plus finement (Bonnefoy et al 2009). L'évolution de la qualité sanitaire de rations composées d'ensilages (herbe ou maïs) et de concentré a été testée séparément en ambiance contrôlée. Aucun risque n'a été montré à une température de $10^{\circ} \mathrm{C}$, alors qu'à 20 et $30^{\circ} \mathrm{C}$ on observe une augmentation de la température dans l'ensilage de maïs et des moisissures dès le deuxième jour. Avec l'ensilage d'herbe, la reprise de fermentations est limitée et il faut attendre le troisième jour pour observer le même résultat qu'avec l'ensilage de maïs.

Les transformations liées à des variations de teneur en MS au sein de l'ensilage dans les auges, de reprise de fermentation ou de mauvaise conservation en général risquent donc de poser des problèmes sanitaires et de toxicité liés aux moisissures, sans être toutefois associés systématiquement à une diminution de l'ingestion et des performances des animaux. La distribution pour plusieurs jours d'une ration «humide» serait ainsi possible sans affecter sa qualité tant que la température n'atteint pas celle d'un plein été.

\section{3 / Substituer des fourrages distribués par du pâturage hivernal}

La simplification de l'affouragement hivernal peut à l'extrême passer par sa suppression quasi-totale en le remplaçant quand c'est possible par du pâturage d'herbe sur pied. L'absence totale de récolte de fourrage pour la période hivernale est cependant rarement pratiquée pour les bovins sous nos climats à saisons marquées. Pour les ovins allaitants, le pâturage hivernal permet de réduire significativement les besoins en stocks de fourrages récoltés et se pratique assez couramment dans l'Ouest et le Centre de la France (Pottier et al 2001), mais avec une forte variabilité de sa mise en œuvre entre les exploitations d'une même zone (Agabriel et al 2011).

Les pratiques d'allongement des périodes de pâturage ne peuvent cependant pas s'envisager sans une contrepartie minimale en termes de performances de production ou de variations d'état corporel. Ces conséquences ont été particulièrement étudiées en production ovine dans des zones de climat océanique avec des hivers doux comme le Montmorillonnais (Ouest du Limousin), ou dans des zones plus sèches à climat méditerranéen en tirant le plus grand parti des parcours et des zones «pastorales» (Molénat et al 2003). Dans ces travaux, les auteurs insistent sur l'organisation raisonnée des systèmes de production pour faire correspondre au mieux les périodes de risques de disette avec les périodes de besoins réduits des troupeaux. Ils insistent également sur l'importance 
Tableau 4. Evolution du poids et de l'état corporel de vaches Charolaises en milieu de gestation conduites en hivernage extérieur avec pâturage hivernal ou en stabulation libre en bâtiment alimentées avec des rations limitées (moyenne par lot, $n=8$ ) (Note et al 2010).

\begin{tabular}{|l|c|c|c|c|c|c|}
\hline & \multicolumn{2}{|c|}{ Début décem bre } & \multicolumn{2}{c|}{$\begin{array}{c}\text { Fin du pâturage hivernal } \\
\text { (mi mars) }\end{array}$} & \multicolumn{2}{|c|}{ Après vêlage } \\
\cline { 6 - 7 } (mi-mai à mi-juin)
\end{tabular}

${ }^{*} E C$ : Note d'Etat Corporel (Agabriel et al 1986).

Au sein d'une même case, les moyennes marquées par des lettres différentes sont significativement différentes.

de gérer la conduite de l'animal par l'estimation de l'état des réserves corporelles de la brebis (Pottier et al 2002).

Avec des bovins allaitants, la réduction de la récolte des fourrages a été associée à l'allongement de la période de pâturage afin de réduire les charges de bâtiments, d'alimentation et de main-d'œuvre qui pèsent sur ces systèmes. A ces impératifs, s'ajoutent désormais davantage d'exigences sur les conditions de l'hivernage extérieur pour préserver le bien-être des animaux, ainsi que sur les conséquences environnementales pour éviter les fuites de polluants par les boues et le ruissellement sur les parcelles.

Seules les conséquences sur les performances animales de l'utilisation d'un stock d'herbe sur pied pour un pâturage hivernal partiel sont abordées ici. Elles permettent d'évaluer l'intérêt zootechnique de ce type de simplification en comparaison d'autres solutions préalablement envisagées qui sont demandeuses d'investissements plus importants.

L'utilisation de stocks d'herbe sur pied, pour accroître la période de pâturage en hiver de vaches taries Charolaises sans dommage sur les parcelles pâturées, a été étudiée à l'Unité Expérimentale des Monts d'Auvergne (Note et al 2010). Cette pratique vise à réduire les récoltes et l'apport de fourrages secs à des vaches taries choisies pour leurs faibles besoins. Trois lots de huit vaches Charolaises taries et au troisième mois de gestation ont été suivis au cours de deux expérimentations indépendantes menées pendant les hivers 2008 et 2009 sur le site de Laqueuille (1000 m d'altitude). Le lot en pâturage hivernal a eu accès du $1^{\text {er }}$ décembre à la mi-mars à une prairie permanente riche en dactyle de 3,7 ha sur laquelle avait été constitué un stock d'herbe sur pied par mise en défend de la parcelle à partir du mois d'aout. Ce stock s'élevait à environ 3,4 t MS/ha en entrée de parcelle. Ce lot disposait d'un abri ouvert sur un parc stabilisé d'hivernage sur copeaux. Mais dans ces conditions de montagne un affouragement minimum est nécessaire lors des périodes de neige. La règle appliquée consistait à apporter du foin lorsque la neige couvrait le sol plus de 48 heures d'affilée. De même, le recours à l'affouragement était appliqué dès lors que les notes d'état corporel de deux vaches du lot étaient inférieures à 1,5 . Les deux autres lots conduits en bâtiment ont été affourragés avec des apports égaux aux besoins d'entretien plus 1 UFL pour l'un et aux besoins d'entretien moins 1 UFL pour l'autre. Ils servaient de témoins pour encadrer les performances attendues du lot expérimental.

Les observations en période hivernale ont porté sur la performance zootechnique et le bilan fourrager en considérant l'évolution de la valeur nutritive de l'herbe offerte. Les adaptations aux conditions climatiques plutôt extrêmes pour ce type de pratique ont fait l'objet d'observations particulières (bien-être animal, choix alimentaire), tout comme l'impact de ce pâturage hors saison de végétation sur la prairie et son potentiel de production. Quatre exclos dans la parcelle pâturée ont ainsi permis de suivre l'évolution de la sénescence de l'herbe tout au long de l'hiver.
Les résultats obtenus montrent que la valeur nutritive de l'herbe offerte au pâturage en hiver est faible et que sa digestibilité estimée par la digestibilité enzymatique pepsine-cellulase, diminue au fil des mois de 58 à $47 \%$. La teneur en matières azotées totales de l'herbe offerte s'est maintenue autour de $12 \%$ le premier hiver et de $9 \%$ le second. Au cours des deux hivers 2008 et 2009 les vaches sont restées au pâturage respectivement 105 et 78 jours et ont été affouragées pendant 56 et 42 jours. Dans le cadre des règles de conduite adoptées, les vaches ont bien supporté les contraintes auxquelles elles ont été soumises et les performances zootechniques n'ont pas été fortement affectées malgré les difficultés de pâturage. Entre décembre et mars, le lot placé en extérieur a maintenu son poids, mais a perdu 0,4 point d'état corporel, les deux autres lots appariés et hivernés normalement gagnant respectivement 13 et $59 \mathrm{~kg}$ de poids vif (dont le fotus), le lot alimenté aux besoins d'entretien moins 1 UFL perdant 0,3 point d'état. Après le vêlage de juin les trois lots ne montrent plus de différences de poids significatives (tableau 4).

Le chargement hivernal cumulé sur les parcelles pâturées est resté assez limité. Il a été estimé à 150 et 210 UGB jour/ha. Dans ces conditions, il n'a pas été noté de conséquences négatives sur la production annuelle ultérieure de la prairie. Entre le fourrage perdu en été (car non récolté) et les prélèvements d'hiver, le bilan fourrager annuel de la parcelle est ainsi variable d'une année à l'autre (respectivement $+1,4$ et $-1,3 \mathrm{t}$ MS) (tableau 5). Il dépend fortement du climat hivernal et des règles d'affoura- 
Tableau 5. Bilans fourragers annuels d'une parcelle conduite en pâturage hivernal et exploitée par un lot $(n=8)$ de vaches Charolaises en gestation (Note et al 2010).

\begin{tabular}{|l|c|c|}
\hline & $2007-2008$ & 2008-2009 \\
\hline Nombre de jours de pâturage & 105 & $78^{*}$ \\
\hline dont nombre de jour affourragés & 56 & 42 \\
\hline $\begin{array}{l}1 \text { = Estimation de la consommation au pâturage } \\
\text { à partir des prélèvements d' herbe (t MS) }\end{array}$ & 4,9 & 2,4 \\
\hline $\begin{array}{l}\text { 2 = Estimation de la récolte non prélevée pour } \\
\text { constituer le stock sur pied (t MS) }\end{array}$ & $-7,5$ & $-6,8$ \\
\hline Bilan de la parcelle (2-1) (t MS) & $-2,6$ & $-4,4$ \\
\hline Economie de fourrage distribué (t MS) & 4,2 & 3,1 \\
\hline
\end{tabular}

${ }^{*}$ Au cours de l'hiver 2008/2009, le pâturage n'a pu commencer qu'en janvier 2009 à cause d'une chute de neige importante et précoce.

gement adoptées. Mais il reste difficile à quantifier et donc à prévoir.

La matière sèche non pâturée en fin d'hiver peut être importante et des essais supplémentaires doivent permettre de mieux l'estimer pour mieux la gérer. Plusieurs facteurs de variation seraient à étudier tels que le rôle de l'importance du stock d'herbe en entrée de pâturage et la capacité plus ou moins importante des espèces de la prairie à être valorisées en hiver. La poursuite de ce programme pendant plusieurs années visera aussi à mieux connaître les évolutions du comportement alimentaire des vaches et des choix de report entre le fourrage sur pied et le fourrage disposé au râtelier. Un tel dispositif pluriannuel permettra enfin de disposer d'une gamme élargie de conditions de températures et d'enneigement.

\section{3 / Simplifier la traite des vaches laitières}

La traite des vaches étant une des activités les plus chronophages et astreignantes, cela fait déjà plusieurs décennies que les éleveurs laitiers comme les chercheurs tentent de la simplifier en agissant sur certaines phase du processus (nettoyage/désinfection des trayons, élimination des premiers jets...) ou sur sa fréquence. Ceci est d'autant plus nécessaire que l'on assiste à une augmentation régulière de la taille des troupeaux laitiers. Toute simplification même minime de la traite entraîne une diminution du temps de travail de l'éleveur. Il existe également, depuis un peu plus de 10 ans, des robots de traite qui permettent de réduire d'environ 2 heures par jour le temps consacré à la traite et son caractère astreignant, avec un investissement financier majoré de 50\% par rapport à une salle de traite clas- sique et un coût de fonctionnement annuel multiplié par deux. Environ $2 \%$ des exploitations bovines laitières françaises sont équipées d'un robot (soit environ 2000 exploitations en 2010), dont l'impact zootechnique est réel : accroissement limité de la production laitière, augmentation importante de la teneur du lait en acides gras libres (lipolyse) et en spores butyriques, etc. (Pomiès et al 2008). Mais s'agissant d'un outil plus que d'une pratique, les conséquences de l'utilisation d'un robot de traite ne seront pas développées dans cet article.

Après avoir présenté les différentes pratiques observées et leurs répercussions en terme de travail, nous concentrerons notre analyse sur les conséquences d'une modification des rythmes de traite sur la production laitière, la composition du lait et la santé, au sens large, des animaux.

\section{1 / Les différentes pratiques}

a) La simplification du processus de traite

Quatre phases de la traite peuvent être réduites ou supprimées afin de gagner du temps : le nettoyage des trayons avant la traite, l'observation des premiers jets de chaque trayon, la désinfection des trayons après la traite et la durée de la traite en elle-même.

Les trois premières n'ont pas de conséquences sur la production laitière et la composition du lait, mais peuvent aboutir à des problèmes sanitaires si la situation initiale du troupeau n'est pas bien maîtrisée. Différents essais (Fourichon et al 1998, Menard et al 1998) ou enquêtes de l'Institut de l'élevage ont montré que la suppression du nettoyage de la mamelle avec des lavet- tes individuelles (environ - $54 \mathrm{~min} / \mathrm{j}$ pour 60 vaches) ou de la désinfection des trayons en fin de traite (environ - $8 \mathrm{~min} / \mathrm{j}$ pour 60 vaches) entraîne généralement une dégradation de la qualité hygiénique du lait (augmentation du nombre de germes, de cellules somatiques et/ou des spores butyriques), pouvant entraîner des pénalités de paiement pour l'éleveur. L'observation des premiers jets dans un bol à fond noir (environ $20 \mathrm{~min} / \mathrm{j}$ pour 60 vaches), en permettant de détecter précocement les mammites cliniques, augmente les chances de guérison par traitement antibiotique et préserve la santé et le bienêtre des animaux. Il faut noter que l'une ou l'autre de ces deux pratiques (observation des premiers jets ou nettoyage des trayons) est nécessaire car elles ont toutes deux un rôle de stimulation mécanique de la mamelle entraînant une décharge d'ocytocine qui, si elle n'a pas lieu, peut rallonger de plusieurs minutes la durée de la traite.

La réduction de la durée de la phase de traite à proprement parler a longtemps été envisagée. Elle est possible depuis peu, grâce à la programmation du seuil de décrochage automatique du faisceau trayeur de certaines installations récentes. Classiquement, la traite prend fin quand le débit de lait trait devient inférieur au seuil de $200 \mathrm{~g} / \mathrm{min}$. Remonter ce seuil à $800 \mathrm{~g} / \mathrm{min}$ permet de raccourcir la traite et de gagner en moyenne $29 \mathrm{~min} / \mathrm{j}$ pour un troupeau de 50 vaches Montbéliarde (Courtot et al 2009). A l'échelle de la semaine, ce réglage modifié est sans effet sur la quantité totale de lait trait. En effet le lait non extrait à la fin d'une traite est recueilli à la suivante. Il n'y a pas non plus d'incidence sur la composition moyenne du lait et sur la santé de la mamelle.

\section{b) La modification des rythmes de traite}

Pour des raisons liées à l'astreinte du travail plus qu'à sa durée, de nombreux éleveurs laitiers sont aujourd'hui tentés de rompre avec la «routine» de traite : 2 traites par jour espacées de 12 heures (10 et 14 heures dans le meilleur des cas), 365 jours par an.

En France, dès les années 1960, des chercheurs ont envisagé de ne traire les vaches que 13 fois par semaine en supprimant la traite du dimanche soir (Labussière et Coindet 1968). Mais cette pratique n'a pas suscité l'intérêt des éleveurs, à une époque où ces derniers cherchaient à produire plus de lait plutôt qu'à disposer de temps libre. Elle a retrouvé un regain d'intérêt dans les années 1990, après l'instauration des quotas laitiers, au sein d'élevages de 
Figure 7. Conséquences de l'intervalle de temps séparant les traites sur la sécrétion apparente (a) et sur la perte de lait (b) chez la vache laitière (d'après Rémond et al 1999).

a)

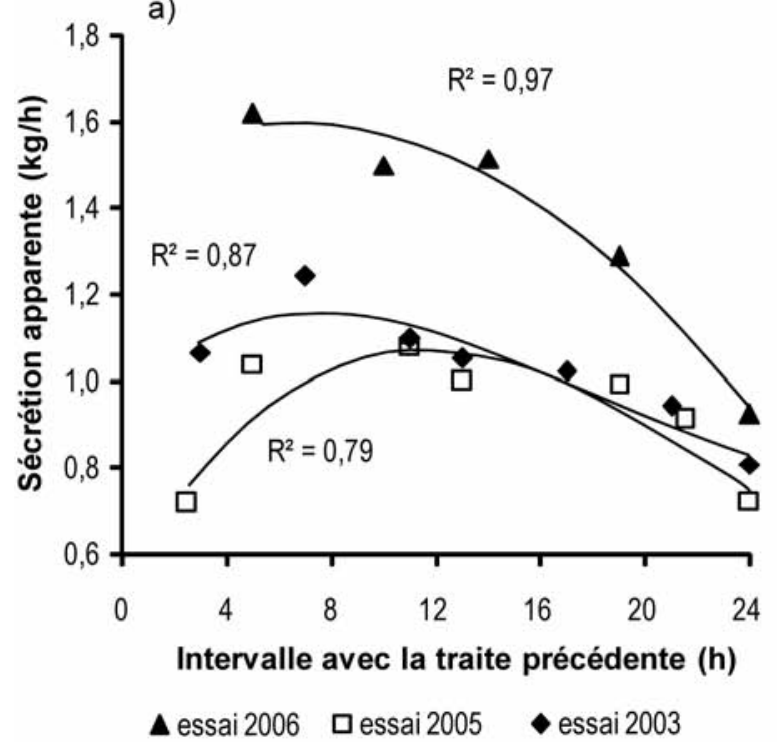

b)

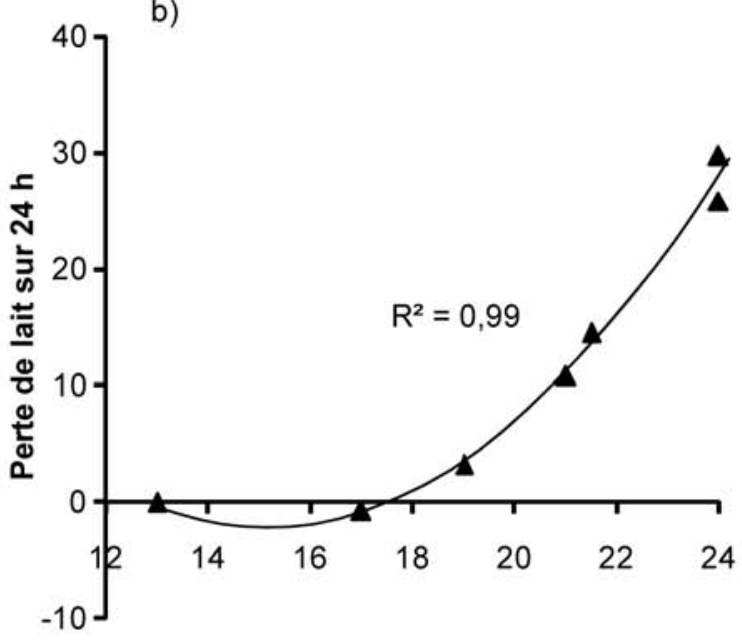

Intervalle entre les traites les plus éloignées (h) taille moyenne n'ayant pas recours à de la main-d'œuvre externe et cherchant à préserver leur vie de famille ; aujourd'hui près de $17 \%$ des éleveurs du Finistère l'appliqueraient (Portier et al 2006).

Dans les années 2000, l'INRA et l'Institut de l'élevage ont étudié au travers de nombreuses expérimentations et enquêtes, les conséquences de la traite des vaches laitières une seule fois par jour : la monotraite. Imaginée pour écrêter le pic de lactation et ainsi réduire la quantité d'aliments concentrés distribuée aux animaux sans risque nutritionnel (Rémond et al 1999), cette pratique s'est rapidement imposée aux éleveurs cherchant à se libérer de l'astreinte de la traite pour une période de quelques jours à quelques mois.

L'INRA a également travaillé sur les conséquences d'horaires de traite atypiques comme la pratique de 3 traites en 2 jours (avec des intervalles de 14 à 18 heures) (Rémond et Boit 1997), ou plus récemment sur les intervalles courts entre les 2 traites de la journée (de 5 à 7 heures) (Rémond et al 2009, Rémond et al 2010). La première pratique, bien que procurant une moindre charge de travail, impose un rythme très irrégulier qui semble difficilement compatible avec le mode de vie des éleveurs. En revanche la seconde, qui permet de positionner les traites en début de matinée et d'après-midi (voire en fin de matinée), devrait être appelée à se développer car elle offre à l'éleveur plus de souplesse dans l'organisation de son travail, de ses loisirs et de sa vie familiale.

Toutes ces pratiques entraînent des périodes où la durée de stockage du lait dans la mamelle s'allonge, avec des conséquences sur le niveau de production laitière et la composition du lait, mais aussi parfois sur d'autres paramètres zootechniques tels que l'ingestion, le poids et l'état corporel, la reproduction, la santé, le comportement et le bien-être animal.

\section{2 / Conséquences de rythmes de traite irréguliers sur la pro- duction laitière}

a) Conséquences à court, moyen et long terme sur le volume de lait produit

Il est classiquement admis que, chez les bovins, la sécrétion horaire de lait par la mamelle est continue durant la dizaine d'heures qui suit la traite. Audelà, le taux de sécrétion apparent décroît sous l'influence de phénomènes mécaniques (capacité de stockage de la mamelle) et hormonaux. Ainsi la traite 3 fois par jour, à des intervalles proches de 8 heures, permet d'augmenter de 10 à $15 \%$ la production laitière d'un troupeau par rapport à une traite biquotidienne classique (Ipema et Benders 1992). A l'inverse, au-delà d'une douzaine d'heures d'intervalle entre traites, la sécrétion horaire tend à diminuer, d'abord progressivement puis plus rapidement (figure 7a, d'après Rémond et al 2009). C'est ce phénomène qui explique les diminutions immédiates de production laitière engendrées par la plupart des pratiques de modification du rythme de traite.

Ainsi, lors de la suppression de la traite du dimanche soir, la perte de lait hebdomadaire varie de moins de $5 \%$ si l'intervalle entre la traite du dimanche matin (généralement retardée par les éleveurs) et celle du lundi matin est réduit à 21 heures (Meffe et al 2003), à $10 \%$ si cet intervalle reste strictement de 24 heures (Pomiès et Rémond 2000).

De même, les éleveurs qui rapprochent les deux traites de la journée sont confrontés à un intervalle long entre la traite de l'après-midi et celle du lendemain matin. Mais ce n'est qu'à partir d'une vingtaine d'heures que les pertes de lait deviennent significatives (figure $7 \mathrm{~b}$, d'après Rémond et al 2009). Ainsi, le passage de 14 heures à près de 17 heures de l'intervalle nocturne entre traites du troupeau de l'INRA de Theix (traite à $14 \mathrm{~h} 20$ et $7 \mathrm{~h} 10$ le lendemain) n'a entraîné aucune diminution de production laitière au cours des 4 semaines suivantes (Rémond et al 2010). Enfin, ces mêmes données montrent qu'un intervalle régulier de $24 \mathrm{~h}$ entre traites, correspondant au cas de la monotraite, conduit à des pertes de lait de 25 à $30 \%$, ce que confirment toutes les études menées dans des conditions similaires (Rémond et Pomiès 2005).

A moyen terme, si ces différentes pratiques sont poursuivies au-delà de quelques semaines après le pic de lactation, la perte de lait (exprimée en $\mathrm{kg} / \mathrm{j}$ ) par rapport à un lot témoin reste relativement constante, voire diminue légèrement. Mais cela se traduit par un accroissement progressif de l'écart de production relatif, que ce soit pour la suppression de la traite du dimanche soir (Labussière et Coindet 1968, Pomiès et Rémond, communication personnelle) ou pour la monotraite (de $-24 \%$ à $-48 \%$ entre la 18 ème et la 44 ème semaine de lactation) (Pomiès et al 2004). 
Figure 8. Variabilité de la perte de lait chez la vache laitière en monotraite, selon la production laitière initiale (mesures sur 237 animaux) (d'après Rémond et Pomiès 2005).

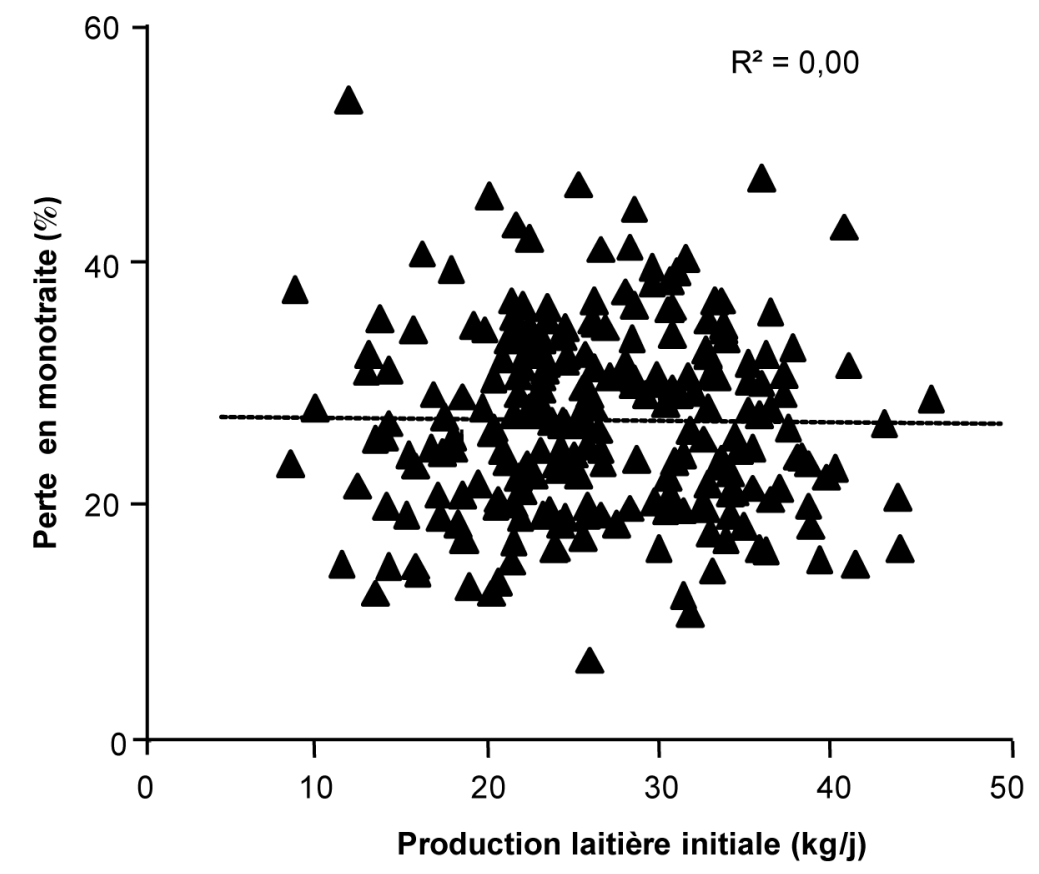

Pour les lactations répétées, seules les conséquences de la monotraite ont été étudiées. Ainsi, le profil des courbes de lactation de vaches en monotraite 3 années successives est analogue à celui des témoins, avec un écart constant d'une lactation à l'autre $(-10,6 \pm$ $0,25 \mathrm{~kg} / \mathrm{j})$ et une perte relative diminuant avec le rang de lactation (de $-46 \%$ pour des primipares à $-37 \%$ pour des vaches en $3^{\text {eme }}$ lactation) (Pomiès et al 2008).

\section{b) Effet rémanent}

Quand les animaux sont à nouveau traits régulièrement 2 fois par jour avec des intervalles de 10 à 14 heures, il n'existe apparemment pas d'effet rémanent de ces pratiques, sauf pour la monotraite. Après une période de monotraite, les vaches ne retrouvent pas le niveau de production d'animaux témoins traits en permanence 2 fois par jour. Cet effet rémanent s'accroît avec la durée de monotraite (de - $3 \%$ après une semaine à - $10 \%$ après 10 semaines) et sa précocité dans la lactation (Rémond et Pomiès 2005). Mais il n'existe pas d'effet rémanent d'une lactation à l'autre, même après 3 lactations successives en monotraite (Pomiès et al 2008).

\section{c) Variabilité individuelle}

La capacité de stockage de la citerne mammaire semble déterminante dans l'aptitude des bovins à supporter des écarts de traite longs (Marnet et Komara 2008). Cette dernière varie avec la race d'après Rémond et Pomiès 2005), et sont très répétables pour un même animal en cours de lactation (Rémond et Pomiès 2007a). Enfin, une étude récente (Guinard-Flament et al 2011) a montré que l'aptitude des vaches à perdre peu de lait lors de grands intervalles de traite et l'aptitude à bien récupérer après retour à des intervalles classiques sont des critères indépendants, sur lesquels une future sélection génétique pourrait s'appuyer.

\section{d) Composition du lait}

L'allongement de l'intervalle entre traites entraîne des modifications de composition du lait qui peuvent avoir une incidence sur le prix payé aux éleveurs. Ainsi, au cours des essais conduits par l'INRA en Auvergne, la monotraite a entraîné une augmentation du taux protéique de $1,4 \mathrm{~g} / \mathrm{kg}$ en moyenne, qui reflète surtout l'amélioration du bilan énergétique des animaux (voir paragraphe 3.3). Les variations de taux butyreux sont, quant à elles, directement et fortement liées à l'intervalle entre traites (figure 9, d'après Rémond et al 2009), qui conduit à une augmentation moyenne de $3,0 \mathrm{~g} / \mathrm{kg}$ pour les lots de vaches en monotraite. Dans le cas des autres pratiques, le mélange de lait issu de traites successives n'entraîne pas de différences de composition du lait de tank (Pomiès et Rémond 2000, Rémond et al 2009). La teneur en acides gras libres, composante principale de la lipo-

Figure 9. Influence de l'intervalle entre traites sur le taux butyreux du lait trait (d'après Rémond et al 2009).

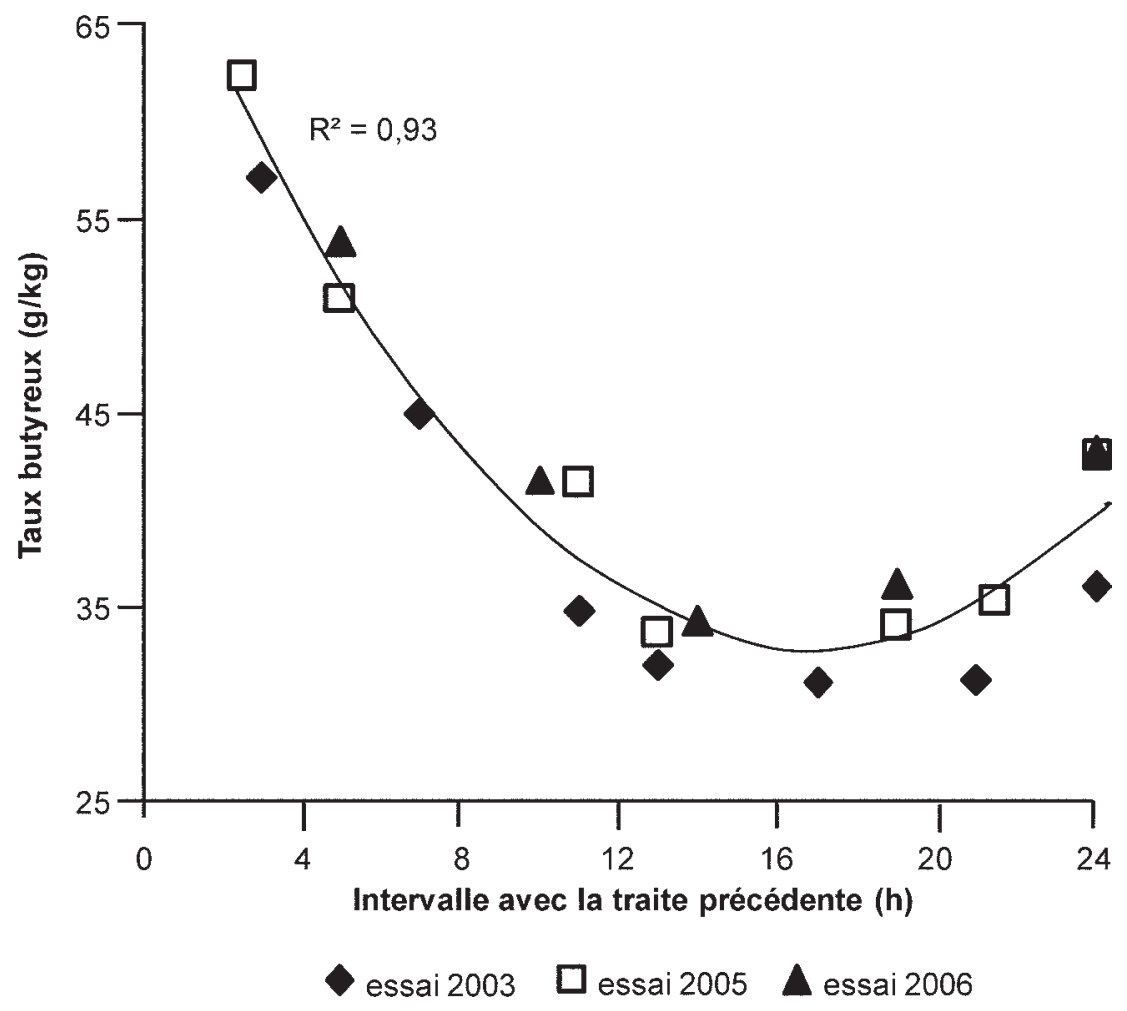


lyse du lait, quant à elle, est corrélée négativement avec l'intervalle entre traites et varie linéairement de 1,15 à $0,38 \mathrm{mEq} / \mathrm{mL}\left(\mathrm{R}^{2}=0,83\right)$ pour des intervalles allant de $2 \mathrm{~h} 30$ à $24 \mathrm{~h}$ (Rémond et al 2009). Ces fortes variations dans la composition du lait posent des problèmes d'homogénéité des fabrications fromagères, en particulier pour les éleveurs qui transforment après chaque traite, comme dans le cas de l'AOP St Nectaire fermier.

\section{3 / Autres conséquences zoo- techniques des modifications du rythme de traite}

\section{a) Ingestion, poids et état corporel}

Parmi les pistes de simplification de la traite, la monotraite est la seule pratique qui entraîne une diminution avérée des quantités de fourrages ingérées, mais ce n'est généralement qu'après 6 à 8 semaines que les vaches commencent à réguler leur ingestion (Rémond et al 2004). Il en résulte, durant cette période où les vaches produisent 25 à $30 \%$ de moins tout en mangeant autant, une augmentation importante du bilan énergétique calculé $(+2$ à $+3 \mathrm{UFL} / \mathrm{j})$, confirmé par des teneurs plasmatiques plus élevées en glucose et plus faibles en acides gras non estérifiés (Rémond et al 1999). Cela se traduit chez les vaches en monotraite par une reprise de poids et d'état corporel plus rapide en début de lactation $(+30 \mathrm{~kg}$ et 0,8 point de note d'état après 12 semaines) (Pomiès et Rémond 2002), qui ne s'observe plus après le pic de lactation, quand les besoins des animaux sont moindres. Par ailleurs, une réduction des apports de concentré chez les vaches en monotraite, souvent envisagée par les éleveurs, se traduit par une baisse de production laitière supplémentaire, équivalente à celle provoquée chez des vaches traites classiquement (Rémond et al 2005).

\section{b) Reproduction et santé}

L'amélioration du bilan énergétique induit par la monotraite en début de lactation se traduit généralement par une reprise de la cyclicité ovarienne et une détection des chaleurs plus précoces, ainsi qu'une diminution de l'intervalle vêlage-insémination fécondante (Rémond et Pomiès 2007b).

Dès que la durée de l'intervalle entre traites dépasse les 20 heures, l'accumulation rapide du lait dans la mamelle provoque une augmentation immédiate, importante ( 2 à 3 fois plus) mais transitoire $( \pm 4$ jours) de la concentration en cellules somatiques du lait (Pomiès et Rémond 2000, Rémond et Pomiès 2007a). Ce phénomène se reproduit toutes les semaines avec la suppression de la traite du dimanche soir, alors que lors du passage en monotraite, au-delà de ces quelques jours, seule une faible différence persiste. En revanche, quand la monotraite est mise en œuvre dès le vêlage et/ou se poursuit plusieurs mois (voire durant toute la lactation), la concentration en cellules somatiques s'accroît régulièrement. Ces niveaux élevés de cellules, ponctuels ou persistants, peuvent être pénalisants pour le paiement du lait aux éleveurs et s'accompagnent parfois de mammites cliniques. En revanche, utilisée l'été avec des animaux pâturant des parcelles éloignées, la monotraite permet de diviser par deux les déplacements et donc de réduire les problèmes de boiteries (Brocard et al 2007).

\section{c) Modifications comportementales et bien-être animal}

Les variations de fréquence des mammites et des boiteries lors de périodes de monotraite prolongées prouvent que cette pratique n'est pas neutre en termes de santé et de bien-être animal. En revanche, de l'avis des éleveurs et des techniciens des fermes expérimentales, le stockage plus long du lait dans la mamelle, induit par les différentes pratiques de modification des rythmes de traite, ne semble pas poser de problèmes aux vaches au-delà des quelques jours d'adaptation (Guéguen et Brocard 2003, Rémond et al 2004), et les animaux manifestent peu de signes d'inconfort ou de comportements particuliers (vocalisations, excitation à l'heure de la traite omise ou retardée, nervosité à la traite suivante...). Néanmoins, certaines études spécifiquement axées sur le bienêtre des vaches en monotraite, tendent à montrer que la pression intra-mammaire occasionnerait une gêne en début de lactation, mais sans augmentation du cortisol plasmatique (Brulé et al 2003, Tucker et al 2007). Elle se traduit au niveau du troupeau par des vaches debout plus tôt avant l'unique traite de la journée, des mamelles plus dures et des écoulements de lait.

\section{Conclusion}

Ce texte a abordé les conséquences de simplifications de pratiques qui peuvent s'anticiper et même se quantifier pour en minimiser les risques. Mais il n'a pas le souhait d'être exhaustif et s'est limité à quelques exemples qui concernent l'affouragement des animaux, la distribution de l'aliment concentré et les pratiques de traite ; autant de postes qui nécessitent un travail important et parfois pénible de la part de l'éleveur. D'autres exemples auraient pu être développés comme la simplification de la conduite de la reproduction en éleva- ge laitier. Il est en effet connu que le suivi des chaleurs nécessite du temps et de la perspicacité. Une réduction plus ou moins longue de l'investissement dans ce suivi journalier pour les repérer peut donc aussi affecter les résultats finaux de l'exploitation, et doit par conséquent être raisonnée. Il n'a pas été fait non plus état d'autres simplifications possibles concernant les pratiques de pâturage comme la réduction du nombre de parcelles pour le pâturage en rotation, ou l'accroissement simultané de la taille des parcelles et des groupes d'animaux qui les utilisent. Enfin, l'effet de l'introduction dans les élevages d'autres équipements de robotique n'a pas non plus été traité ici.

La simplification du travail d'affouragement ou d'alimentation peut se mettre en œuvre de façons diverses depuis la mécanisation complète de la distribution jusqu'à sa réduction maximale par l'augmentation des séquences pâturées. Les gains de temps et la réduction de la pénibilité du travail qui s'en suivent sont alors très différents et dépendent bien évidemment de la manière dont ces modifications peuvent s'appliquer sur le terrain. La taille et l'organisation spatiale des bâtiments et des parcelles sont par exemple des facteurs qui vont fortement interférer. Nous avons montré, sur un large panel d'exemples envisageant diverses productions et diverses solutions techniques, que ces pratiques simplifiées d'alimentation doivent s'envisager en tenant compte de la nature des aliments (fourrages, aliments concentrés) et des types d'animaux qui reçoivent les rations. Correctement mises en œuvre elles ne modifient peu ou pas les performances d'animaux à niveau de production modéré ce qui illustre la très grande «flexibilité alimentaire» des ruminants vis-à-vis des modalités d'affouragement.

Les distributions simplifiées conduisent les animaux à mobiliser leurs capacités d'adaptation que ce soit à court terme ou sur une plus grande période, et à utiliser si nécessaire leur capacité de rebond compensateur. Plasticité et robustesse des animaux selon la définition de Blanc et al (2010), sont des qualités nécessaires à la mise en œuvre de pratiques simplifiées, et les exemples choisis nous ont permis de rappeler les résultats génériques déjà publiés dans ce domaine. La plasticité du comportement d'ingestion permet en premier lieu de moduler le nombre et la durée des repas et/ou de gérer au mieux les choix entre des aliments proposés soit conjointement soit de façon alternée. La plasticité des fonctions physiologiques s'illustre dans le maintien de l'utilisation digestive des rations lors de séquences alternées extrêmes de 3 à 
4 jours de foin suivies de paille. Dans ce cas la plasticité s'exprime aussi par des phases très courtes et répétées de mobilisation - reconstitution des réserves lipidiques qui ne se mesurent pas aisément avec les méthodes actuellement disponibles. La capacité de mobilisation et de reconstitution des réserves corporelles lipidiques est une qualité requise et presque nécessaire pour tamponner les à coups nutritionnels et les baisses momentanées des apports énergétiques (Agabriel et Petit 1987). On sait que les performances globales à l'échelle du cycle de production ou de la carrière sont peu modifiées grâce à ces capacités de compensation lorsqu'arrivent des périodes d'alimentation pléthorique.

La plasticité de la fonction de lactation est sans doute plus faible puisqu'une modification des conditions d'extraction du lait se traduit directement par des conséquences quantifiables sur la lactation en cours.
Les simplifications de pratiques permettent aux éleveurs d'envisager le maintien de leurs objectifs de production, même lorsque ceux-ci sont élevés avec des vaches laitières, ou des taurillons à l'engrais. Mais les troupeaux allaitants bovins et ovins sont les plus concernés par les simplifications des pratiques d'affouragement. Leur agrandissement continu pousse d'ailleurs les éleveurs à modifier leurs pratiques pour les rendre mieux adaptées aux manipulations de grands groupes. Ainsi la simplification risque encore d'accroître la variabilité des réponses individuelles au sein des groupes qui reste mal connue car mal renseignée. Un indicateur même grossier des performances individuelles au sein des groupes devient nécessaire. L'éleveur qui disposera de cette information pourra juger la réponse de ses animaux sous l'effet d'une modification choisie de pratiques. En ce sens, les développements de matériels d'élevage de précision et de monitoring des fonctions, permettront à l'avenir de prendre davantage de risques dans la simplification car l'information sur les conséquences en termes de performances zootechniques sera plus rapidement disponible.

Au final, la simplification des pratiques peut être vécue de manière très différente par les éleveurs. Tous s'accordent sans doute sur la réduction de la pénibilité du travail que les simplifications par mécanisation peuvent entraîner, mais la distanciation qu'elles risquent d'engendrer vis-à-vis des animaux peuvent être parfois aussi mal vécue. Les réactions peuvent être très diverses selon qu'elles sont considérées comme plus ou moins risquées. C'est chaque éleveur individuellement qui choisit les modifications des pratiques qu'il veut appliquer et c'est alors qu'il peut les évaluer, mais comme toute innovation, un temps pour l'apprentissage est bien souvent nécessaire.

\section{Références}

Agabriel J., Petit M., 1987. Recommandations alimentaires pour les vaches allaitantes. Bull. Tech. C.R.Z.V., Theix, INRA, 70, 153166.

Agabriel J., Giraud J.M., Petit M., 1986. Détermination et utilisation de la note d'état d'engraissement en élevage allaitant. Bull. Tech. CRZV Theix, INRA, 66, 43-50

Agabriel J., Delaby L., Pottier E., 2011. Raisonner et ajuster au mieux le besoin et l'utilisation des fourrages conservés. Fourrages, 205, 25-34.

Bastien D., Chaigneau F., Molle J., 2009. Réduction du temps de travail d'alimentation en bâtiment des bovins viande : distribution des rations trois fois par semaine. Renc. Rech. Rum., 16, 183-186.

Baumont R., Dulphy J.P., Demarquilly C., 1997. Maximiser l'ingestion de fourrages conservés. Renc. Rech. Rum., 4, 57-64.

Blanc F., Dumont B., Brunschwig G., Bocquier F., Agabriel J., 2010. Robustesse, flexibilité, plasticité : des processus adaptatifs révélés dans les systèmes d'élevage extensifs de ruminants. In : Robustesse, rusticité, flexibilité, plasticité, résilience,... les nouveaux critères de qualité des animaux et des systèmes d'élevage. Sauvant D., Perez J.M. (Eds). Dossier, INRA Prod. Anim., 23, 65-80.

Bonnefoy J.C., Boudra H., Doreau M., 2009. Réduction de la fréquence de distribution des ensilages : incidence sur la qualité des rations Renc. Rech. Rum., 16, 63.

Bonnefoy J.C., Chaucheyras-Durand F., Anglard F., Sindou J., Vedrine A., 2010. Effets de la distribution de la ration 2 fois par semaine sur les performances des vaches laitières en production, et efficacité d'un additif pour ensilage sur la stabilité de la ration. Renc. Rech. Rum., 17, 327.

Brocard V., Portier B., Le Meur D., Porhiel J.Y., Lopez C., 2007. Traire les vaches une fois par jour ou grouper les vêlages : deux techniques pour réduire le travail d'astreinte en élevage. Renc. Rech. Rum., 14, 393-396.

Brulé A., Brocard V., Portier B., Racine V., 2003. Effets de la réduction de la fréquence de traite sur le bien être de la vache laitière. Renc. Rech. Rum., 10, 77-80.

Cournut S., Chauvat S., 2012. L'organisation du travail en exploitation d'élevage : analyse de 630 Bilans Travail réalisés dans huit filières animales. In : Numéro spécial, Travail en élevage. (Eds). INRA Prod. Anim., 25, 101-112.

Courtot L., Dupuits G., Chapuis D., 2009. Réduire le temps de traite par la dépose précoce du faisceau trayeur, en race Montbéliarde. Renc. Rech. Rum., 16, 196.

Farrié J.P., Haurez Ph., Chaigneau F., Joulie A., Renon J., 2004. Simplification de l'alimentation hivernale dans les grands troupeaux d'élevage bovin allaitant. Renc. Rech. Rum., 11, 137-140.

Faverdin P., Delagarde R., Delaby L., Meschy F., 2007. Alimentation des vaches laitières. In : Alimentation des bovins, ovins et caprins. Besoin des animaux. Valeurs des Aliments. Tables INRA 2007. Editions Quae, Versailles, France, 23-56.

Favreau A., Ginane C., Baumont R., 2010. Feeding behaviour of sheep fed lucerne $v$. grass hays with controlled posting-ingestive consequences. Animal, 4, 1368-1377.

Fourichon C., Bareille N., Seegers H., Beaudeau F., 1998. Survenue et expression des mammites cliniques et subcliniques en troupeaux bovins laitiers : facteurs de risque liés aux pratiques de traite. Renc. Rech. Rum., 5, 347 .

Friggens N.C., Sauvant D., Martin O., 2010. Towards operational definitions of robustness that rely on biology: nutrition. In : Robustesse, rusticité, flexibilité, plasticité, résilience,... les nouveaux critères de qualité des animaux et des systèmes d'élevage. Sauvant D., Perez J.M., (Eds). Dossier, INRA Prod. Anim., 23, 43-51.

Ginane C., Baumont R., Duncan A.J., 2009. Diet learning through post-ingestive consequences in sheep: the case of starch and casein variously combined in the same foods. Animal, $3,135-142$.

Grenet N., Haurez Ph., Billant J., Imbert F., 1997. Simplification de l'alimentation hivernale en troupeaux allaitants par distribution alternée des fourrages. Renc. Rech. Rum., 4, 148.

Guéguen L., Brocard V., 2003. Une traite par jour en élevage bovin : recueil de l'expérience de 120 éleveurs français. Renc. Rech. Rum., 10,114 .

Guinard-Flament J., Gallard Y., Larroque H., 2011. Lactose in blood plasma and the ability of dairy cows to tolerate once-daily milking in terms of milk loss and milk recovery. J. Dairy Sci., 94, 3446-3454.

Hoch T., Begon C., Cassar-Malek I., Picard B., Savary-Auzeloux I., 2003. Mechanisms and consequences of compensatory growth in ruminants. INRA Prod. Anim., 16, 49-59.

Hostiou N., Fagon J., 2012. Simplification des conduites d'élevage : analyse transversale des pratiques mises en œuvre dans les filières herbivores et granivores. In : Numéro spécial, Travail en élevage. INRA, Prod. Anim., 25, 127-140.

Ingrand S., Agabriel J., 1997. Typology of the evolution of intake by Charolais cows around calving. Animal Sci., 65, 361-371.

INRA, 2007. Alimentation des bovins, ovins et caprins. Besoins des animaux. Valeurs des aliments. Tables INRA 2007. Editions QUAE, Paris, France, 307p.

INRAtion, 2008. Logiciel de rationnement des Ruminants -. In : Agabriel J., Champciaux P., Espinasse C. (Eds). INRAtion, CNERTA, Dijon, France, Version 4.0. 
Ipema A.H., Benders E., 1992. Production, duration of machine-milking and teat quality of dairy cows milked 2, 3 or 4 times daily with variable intervals. In: Proceedings of the international symposium on prospects for automatic milking. Wageningen, Netherlands, USA, 244-252.

Jarrige R., Dulphy J.P., Faverdin P., Baumont R., Demarquilly C., 1995. Activités d'ingestion et de rumination In: Jarrige R., Ruckebusch Y., Demarquilly C., Farce M.H., Journet M. (Eds). Nutrition des Ruminants domestiques, INRA Editions, 123-181.

Keane M.G., Drennan M.J., 2006 Comparison of supplementary concentrate levels with grass silage, separate or total mixed ration feeding, and duration of finishing in beef steers. Livest. Sci. 103, 169-180.

Kentzel M., 2010.Référentiel travail en élevages bovins viande. Réseau mixte technologique travail en élevage synthèse de 170 Bilans Travail. Idele (Ed), Paris, France, 40 p.

Labussière J., Coindet J., 1968. Effets de la suppression de la traite du dimanche soir, chez les bovins de race Française Frisonne Pie Noire. Ann. Zoot., 17, 231-244.

Lassalas J., Agabriel J., Petit M., 1997. Distribution à l'échelle de la semaine d'une ration foin/paille à des vaches allaitantes. Renc. Rech. Rum., 4, 149.

Marnet P.G., Komara M., 2008 Management systems with extended milking intervals in ruminants: regulation of production and quality of milk. J. Dairy Sci., 86, 47-56.

Meffe N., Tache C., Gaudin V., Marnet P.G., 2003. Suppression d'une traite par semaine pendant toute la lactation chez les vaches laitières à haut potentiel : effets zootechniques et caractéristiques physico-chimiques du lait. Renc. Rech. Rum. 10, 85-88.

Ménard J.L., Gaudin V., Billon P., 1998. Hygiène des trayons avant la traite : temps de préparation et coûts selon différentes techniques recommandées. Renc. Rech. Rum., 5, 340.

Mialon M.M., Martin C., Garcia F., Menassol J.B., Dubroeucq H., Veissier I., Micol D., 2008. Effects of the forage-to-concentrate ratio of the diet on feeding behaviour in young Blond d'Aquitaine bulls. Animal, 2, 1682-1691.

Molenat G., Dureau R., Fabre P., Lambertin M., 2003. Herbage used by the transhumant sheep flocks from Crau. The multiple dimensions of pasture and forage management. Fourrages, 437-461.

Normand J., Brouard S., 2001, Agneaux en bergerie, réussir la finition, Institut de l'élevage. In : Pottier E. (Ed), 25-28.

Normand J., Pottier E., Sagot L., Dobbels M., Le Pichon D., 2003. Incidence du mode de distribution des protéagineux sur les performances zootechniques des agneaux en bergerie. Renc. Rech. Rum., 10, 373-376.
Note P., Egal D., Castellan E., D'hour P., 2010. Utilisation hivernale d'un stock d'herbe sur pied par des bovins au pâturage. Renc. Rech. Rum., 17, 62.

Petit M. 1988. Alimentation des vaches allaitantes. In : Jarrige R., Alimentation des bovins ovins et caprins. INRA (Ed), Paris, France, 159-184.

Petit M., Agabriel J., 1993. Etat corporel des vaches Charolaises : signification, utilisation pratique et relations avec la reproduction. INRA Prod. Anim., 6, 311-318.

Pomiès D., Rémond B., 2000. Omission d'une ou deux traites par semaine sur des vaches laitières en milieu de lactation. Renc. Rech. Rum., 7, 128.

Pomiès D., Rémond B., 2002. La traite des vaches laitières une fois par jour pendant l'ensemble de la lactation : conséquences sur les performances zootechniques et la qualité du lait. Renc. Rech. Rum., 9, 195-198.

Pomiès D., Rémond B., Pradel P., 2004 Performances des vaches laitières et qualité du lait lors de la monotraite et après retour à 2 traites par jour, en fonction de la durée de cette pratique et du stade de lactation des animaux. Renc. Rech. Rum., 11, 225-228.

Pomiès D., Martin B., Chilliard Y., Pradel P. Rémond B., 2007. Once-a-day milking of Holstein and Montbéliarde cows for seven weeks in mid-lactation. Animal, 1, 1497 1505 .

Pomiès D., Marnet P.G., Cournut S., Guinard-Flament J., Rémond B., 2008. Les conduites de traite simplifiées en élevage laitier : vers la levée de l'astreinte biquotidienne. In : Numéro spécial, 20 ans de recherches en productions animales à l'INRA. Charley B. Herpin P., Perez J.M. (Eds). INRA Prod. Anim., 21, 59-70.

Portier B., Chénais F., Brocard V., 2006. Traire une fois par jour toute l'année : un revenu équivalent à certaines conditions. Cap Elevage, 6, 10-13.

Pottier E., D'Hour P., Havet A., Pelletier P 2001. Extending the grazing season for suckling herds. Fourrages, 287-310.

Pottier E., Normand J, Sagot L., Le Pichon D., Dobbels M., 2002, Comportement alimentaire de l'agneau en bergerie en finition : des pistes pour simplifier les conduites, Renc. Rech. Rum., 9, 287-290

Rémond B., Boit M.P., 1997. Effects of milking three times in 2 days for 3 weeks in early lactation or in the declining phase on milk production in primiparous and multiparous dairy cows. Ann. Zootech., 46, 339-348.

Rémond B., Pomiès D., 2005. Once-daily milking of dairy cows: a review of recen French experiments. Anim. Res., 54, 427-442.

Rémond B., Pomiès D., 2007a. Once-daily milking of Holstein cows for one-week decreases milk yield by twenty-five percent without any carry-over effect. Livest. Sci., 110, $192-$ 195.

Rémond B., Pomiès D., 2007b. La traite des vaches laitières une fois par jour : conséquences sur leur production, leur bien-être, leur reproduction et leur santé. Bull. GTV, 40, 8390

Rémond B., Coulon J.B., Nicloux M., Levieux D., 1999. Effect of temporary oncedaily milking in early lactation on milk production and nutritional status of dairy cows. Ann. Zootech., 48, 341-352.

Rémond B., Pomiès D., Dupont D., Chilliard Y., 2004. Once-a-day milking of multiparous Holstein cows throughout the entire lactation: milk yield and composition, and nutritional status. Anim. Res., 53, 201-212.

Rémond B., Pomiès D., Pradel P., 2005. Effet de la monotraite des vaches laitières sur leur production, selon le niveau de distribution d'aliments concentrés. Renc. Rech. Rum., 12, 229-232.

Rémond B., Pomiès D., Julien C., GuinardFlament J., 2009. Performance of dairy cows milked twice daily at contrasting intervals. Animal, 3, 1463-1471.

Rémond B., Pomiès D., Bonnefoy J.C. Rudel S., 2010. Un écart de sept heures entre les deux traites de la journée ne modifie pas la production laitière de vaches en milieu de lactation. Renc. Rech. Rum., 17, 261.

Reyne Y., Garambois X., 1977. Utilisation du pois potager comme source d'azote dans les mélanges fermiers pour l'engraissement des agneaux. INRA-ENSA, Montpellier, France.

Schutz, J.S., Wagner, J.J., Neuhold, K.L., Archibeque, S.L., Engle, T.E., 2011. Effect of feed bunk management on feedlot steer intake. Prof. Anim. Sci., 27, 395-401.

Schwartzkopf-Genswein K.S., Beauchernin K.A., McAllister T.A., Gibb D.J., Streeter M., Kennedy A.D., 2004. Effect of feed delivery fluctuations and feeding time on ruminal acidosis, growth performance, and feeding behavior of feedlot cattle. J. Anim. Sci. 82, 3357 3365 .

Schwartzkopf-Genswein K.S., Hickman D.D., Shah M.A., Krehbiel C.R., Genswein B.M.A., Silasi R., Gibb D.G., Crews D.H., McAllister T.A., 2011. Relationship between feeding behavior and performance of feedlot steers fed barley-based diets. J. Anim. Sci., 89, 1180-1192.

Soto-Navarro S.A., Krehbiel C.R., Duff G.C., Galyean M.L., Brown M.S., Steiner R.L., 2000. Influence of feed intake fluctuation and frequency of feeding on nutrient digestion, digesta kinetics, and ruminal fermentation profiles in limit-fed steers. J. Anim. Sci., 78, 2215 2222 .

Tucker C.B., Dalley D.E., Burke J.L., Clark D.A., 2007. Milking cows once daily influences behavior and udder firmness at peak and mid lactation. J. Dairy Sci., 90, 1692-1703. 


\section{Résumé}

Les simplifications des pratiques telles que les modifications des modes et rythmes de distribution de l'alimentation et de la traite, influencent les performances zootechniques des ruminants. La distribution de l'alimentation hivernale par remorque mélangeuse qui broie partiellement les brins longs conduit à des niveaux d'ingestion d'autant plus élevés que les fourrages utilisés sont grossiers. La distribution séparée d'aliment concentrés de différentes natures à des agneaux à l'engrais ne modifie que peu les quantités et proportions moyennes ingérées d'un mélange et n'affecte pas la croissance. Mais la distribution d'ensilage une fois tous les deux jours ou trois fois par semaine, et l'alternance de la distribution des composants de la ration sur la semaine, sont des pratiques qui conduisent à une moindre utilisation nutritive des aliments de la ration. L'utilisation de ces techniques est possible mais limitée à des animaux modérément productifs. La monotraite des vaches réduit la production laitière de 10 à $\mathbf{3 0} \%$ avec un effet rémanent qui est d'autant plus important qu'elle est appliquée tôt dans la lactation et de façon prolongée. C'est une pratique qui entraîne parallèlement une diminution des quantités de fourrages ingérées. Ainsi l'animal peut mobiliser la plasticité du comportement d'ingestion et des processus digestifs et métaboliques pour s'adapter à des rythmes de distribution d'alimentation très variés sans modifier les lois de réponses aux apports alimentaires. Cette capacité d'adaptation est une caractéristique forte des ruminants sur lequel l'éleveur peut s'appuyer pour aménager son travail selon ses contraintes personnelles. En revanche, la plasticité de la lactation est moindre puisqu'une modification des conditions d'extraction du lait se traduit par des conséquences sur la lactation en cours, mais sans effet à l'échelle de la carrière de la vache. La modification des pratiques se raisonne donc toujours au regard des capacités d'adaptation des animaux qui nécessitent d'être mieux quantifiées par les travaux de recherches à venir.

\section{Abstract \\ Consequences of management simplifications on animal performance: the example of feed distribution and milking in cows}

Practice simplifications such as changes in patterns and rhythms of food distribution and milking, influence ruminant performance. The daily distribution of the ration by a mixer wagon which partially grinds long strands, leads to intake levels even higher than when coarse fodders are used. Separate distribution of concentrates of various types to fattening lambs only slightly changes the total intake and the average proportions of the mixture and does not affect growth. But the distribution of silage once every two days or three times a week, or the alternation of the distribution of diet components during the week, are practices that lead to decreased nutritional values of the diet's foods. The use of these techniques is possible, but limited to low / moderate productive animals.

Milking once a day reduced milk production from 10 to $30 \%$ with a residual effect, which increased with the duration of application and lactation. It caused a parallel decrease in the amount of forage ingested. Thus the animal can mobilize the plasticity of the digestive processes and the metabolic rhythms to adapt to very different changes in diet distribution without upsetting the laws of generic responses known. Intake behaviours are also very adaptable and deployable. This is a strong feature of ruminants with moderate requirements on which the farmer can rely. The plasticity of lactation is lower since a change in the conditions of milk extraction modifies the current production, but has no effect on the scale of the cow's career. Changing practices must be thought of in terms of adaptability of animals therefore requiring further research.

AGABRIEL J., FARRIE J.-P., POTTIER E., NOTE P., POMIES D., 2012. Conséquences zootechniques de simplifications de pratiques : exemples de la distribution des aliments et de la traite des vaches. In : Numéro spécial, Travail en élevage. Hostiou N., Dedieu B., Baumont R. (Eds). INRA Prod. Anim., 25, 141-158. 